Review Article

\title{
Noninvasive Brain Stimulations for Unilateral Spatial Neglect after Stroke: A Systematic Review and Meta-Analysis of Randomized and Nonrandomized Controlled Trials
}

\author{
Flávio Taira Kashiwagi, ${ }^{1}$ Regina El Dib, ${ }^{2}$ Huda Gomaa, ${ }^{3}$ Nermeen Gawish, ${ }^{3}$ \\ Erica Aranha Suzumura, ${ }^{4}$ Taís Regina da Silva, ${ }^{1}$ Fernanda Cristina Winckler, ${ }^{1}$ \\ Juli Thomaz de Souza, ${ }^{2}$ Adriana Bastos Conforto $\mathbb{D D}^{5}{ }^{5}$ Gustavo José Luvizutto ${ }^{(D)}{ }^{6}$ \\ and Rodrigo Bazan ${ }^{1}$ \\ ${ }^{1}$ Neurology Department, Botucatu Medical School, Universidade Estadual Paulista (UNESP), Botucatu, SP, Brazil \\ ${ }^{2}$ Science and Technology Institute, Universidade Estadual Paulista (UNESP), São José dos Campos, SP, Brazil \\ ${ }^{3}$ Department of Pharmacy, Tanta Chest Hospital, Tanta, Egypt \\ ${ }^{4}$ Research Institute, Hospital do Coração (HCor), São Paulo, SP, Brazil \\ ${ }^{5}$ Neurostimulation Laboratory, University of São Paulo (USP), São Paulo, SP, Brazil \\ ${ }^{6}$ Department of Applied Physical Therapy, Federal University of Triângulo Mineiro (UFTM), Uberaba, MG, Brazil
}

Correspondence should be addressed to Gustavo José Luvizutto; gluvizutto@fmb.unesp.br

Received 7 September 2017; Accepted 15 April 2018; Published 28 June 2018

Academic Editor: Michele Fornaro

\begin{abstract}
Copyright ( 2018 Flávio Taira Kashiwagi et al. This is an open access article distributed under the Creative Commons Attribution License, which permits unrestricted use, distribution, and reproduction in any medium, provided the original work is properly cited.
\end{abstract}

Background. Unilateral spatial neglect (USN) is the most frequent perceptual disorder after stroke. Noninvasive brain stimulation (NIBS) is a tool that has been used in the rehabilitation process to modify cortical excitability and improve perception and functional capacity. Objective. To assess the impact of NIBS on USN after stroke. Methods. An extensive search was conducted up to July 2016. Studies were selected if they were controlled and noncontrolled trials examining transcranial direct current stimulation (tDCS), repetitive transcranial magnetic stimulation (rTMS), and theta burst stimulation (TBS) in USN after stroke, with outcomes measured by standardized USN and functional tests. Results. Twelve RCTs (273 participants) and 4 non-RCTs (94 participants) proved eligible. We observed a benefit in overall USN measured by the line bisection test with NIBS in comparison to sham (SMD $-2.35,95 \%$ CI $-3.72,-0.98 ; p=0.0001$ ); the rTMS yielded results that were consistent with the overall meta-analysis (SMD $-2.82,95 \%$ CI $-3.66,-1.98 ; p=0.09$ ). The rTMS compared with sham also suggested a benefit in overall USN measured by Motor-Free Visual Perception Test at both $1 \mathrm{~Hz}$ (SMD 1.46, 95\% CI 0.73, 2.20; $p<0.0001$ ) and $10 \mathrm{~Hz}$ (SMD 1.19, 95\% CI 0.48, 1.89; $p=0.54$ ). There was also a benefit in overall USN measured by Albert's test and the line crossing test with $1 \mathrm{~Hz}$ rTMS compared to sham (SMD 2.04, 95\% CI 1.14, 2.95; $p<0.0001$ ). Conclusions. The results suggest a benefit of NIBS on overall USN, and we conclude that rTMS is more efficacious compared to sham for USN after stroke.

\section{Background}

Stroke is the second leading cause of death worldwide and the primary cause of chronic disability in adults [1]. In the United States, it is the fourth leading cause of death overall [2]. Among people who survive a stroke, unilateral spatial neglect (USN) is the most frequent disorder for right hemisphere lesions [3].

The incidence of USN varies widely from $10 \%$ to $82 \%$ $[4,5]$. USN is characterized by the inability to report or respond to people or objects presented on the side contralateral to the lesioned side of the brain and has been associated 
with poor functional outcomes and long stays in hospitals and rehabilitation centers [6].

Pharmacological interventions such as dopamine and noradrenergic agonists or procholinergic treatment have been used in people affected by USN after stroke, but the evidence derived from a Cochrane systematic review that included only two available RCTs was very low and inconclusive [7].

Other nonpharmacological rehabilitation techniques have been explored for USN with the aim to facilitate the recovery of perception and behavior, which include right half-field eye-patching [8], mirror therapy [9], prism adaptation [10], left-hand somatosensory stimulation with visual scanning training [11], contralateral transcutaneous electrical nerve stimulation and optokinetic stimulation [12], trunk rotation [13], repetitive transcranial magnetic stimulation [14], galvanic vestibular stimulation [15], and dressing practice [16]. However, their results do not support the use of these techniques in isolation for improvement of secondary outcomes such as performance and sensorimotor functions, activities of daily living (ADLs), or quality of life $[9,14,17]$.

Noninvasive brain stimulations (transcranial direct current stimulation (tDCS) and repetitive transcranial magnetic stimulation (rTMS)) have already shown their ability to modify cortical excitability [18]. tDCS is a noninvasive method used to modulate cortical excitability by applying a direct current to the brain that is less expensive than repetitive transcranial magnetic stimulation (rTMS). The latter is an electric current that creates magnetic fields that penetrate the brain and can modulate cortical excitability by decreasing or increasing it and potentially improve perceptual and cognitive abilities $[19,20]$.

A previous Cochrane systematic review summarized results about the effects of tDCS versus control (sham/any other intervention) on activities of daily living (ADLs) among stroke survivors. The authors included 32 randomized controlled trials (RCTs) and concluded that tDCS might enhance ADLs, but upper and lower limb function, muscle strength, and cognitive abilities should be further explored [21]. Another Cochrane systematic review assessed the efficacy of repetitive transcranial magnetic stimulation (rTMS) compared to sham therapy or no therapy for improving function in people with stroke. The 19 included trials showed that rTMS was not associated with a significant increase in ADLs or in motor function; therefore, the authors do not support the use of rTMS for the treatment of stroke, and they plan to complete further trials to confirm their findings [22].

Previous reviews were, however, limited in that they did not include non-RCT studies nor did they evaluate the newest noninvasive brain stimulation - theta burst. We therefore conducted a systematic review of RCT and non-RCT studies that assessed the impact of tDCS, rTMS, and TBS for unilateral spatial neglect after stroke.

\section{Methods}

We adhered to methods described in the Cochrane Handbook for Intervention Reviews [23]. Our reporting also adheres to the Preferred Reporting Items for Systematic
Reviews and Meta-Analyses (PRISMA) [24] and MetaAnalysis of Observational Studies in Epidemiology (MOOSE) statements [25].

2.1. Eligibility Criteria. The eligibility criteria are as follows:

(1) Study designs: RCTs, quasi-RCTs, and non-RCTs

(2) Participants: adults over 18 years of age, regardless of gender and the duration of illness or severity of the initial impairment, with USN after any type of stroke diagnosis (ischemic or intracranial hemorrhage) measured by clinical examination or radiographically by computed tomography (CT) or magnetic resonance imaging (MRI), regardless of whether they were included after evaluation by standardized USN tests.

(3) Interventions: any noninvasive brain stimulations such as tDCS, rTMS, and including theta burst (continuous TBS (cTBS) or intermittent theta burst (iTBS)) (we considered evaluating both the different types of stimulations (i.e., cathodal tDCS versus anodal tDCS versus dual tDCS) and types of frequency (i.e., high-frequency versus low frequency))

(4) Comparators: interventions were to be compared against sham stimulation or any conventional stroke rehabilitation (e.g., pharmacological therapy or nonpharmacological therapy such as right half-field eyepatching, mirror therapy, prism adaptation, left-hand somatosensory stimulation, and visual scanning training or other conventional treatment)

We also considered noninvasive brain stimulations as an adjunct to any type of conventional stroke rehabilitation.

(5) Outcomes:

(i) Overall USN measured by any paper-andpencil tests, such as the line cancellation task [26], the line bisection test [27], or the star cancellation test [28], and by any validated specific instrument, such as the Catherine Bergego Scale [29], and the Behavioral Inattention Test [30]

(ii) Disability in neurological and functional abilities as measured by any validated specific instrument, such as the National Institutes of Health Stroke Scale and the Modified Rankin Scale [31], the box and block test [32], or the Fugl-Meyer Assessment [33] after treatment and over the long term

(iii) Daily life functions as measured by any validated measurement scale, such as the Barthel index [31]

(iv) Number of reported falls as measured by diaries of falls, by the Morse Fall Scale [34], or by the Hendrich II Fall Risk Model [35] after treatment and over the long term 
(v) Balance as measured by the Berg Balance Scale, the balance subscale of the Fugl-Meyer test, and the Postural Assessment Scale for Stroke Patients [36] after treatment and over the long term

(vi) Depression or anxiety as measured by the Beck Depression Inventory, the Hospital Anxiety and Depression Scale, Symptom Checklist-90 (SCL-90), and the Hamilton Depression Rating Scale [37] after treatment and over the long term

(vii) Evaluation of poststroke fatigue by the Fatigue Severity Scale [38] after treatment and over the long term

(viii) Quality of life (however defined by the study authors) after treatment and over the long term

(ix) Adverse events (e.g., euphoria, hallucinations, orthostatic hypotension, nausea, insomnia, dizziness, and syncope) after treatment and over the long term

(x) Death

2.2. Data Source and Searches. We searched MEDLINE (OvidSP) (1966 to July 2016), EMBASE (OvidSP) (1980 to July 2017), the Cochrane Central Register of Controlled Trials (CENTRAL) (The Cochrane Library, 2017, issue 7), CINAHL (1961 to July 2017), and Latin-American and Caribbean Center on Health Sciences Information (LILACS) (from 1982 to July 2017) without language restrictions. The date of the most recent search was 26 July 2017. All searches were conducted with the assistance of a trained medical librarian. We also searched the reference lists of relevant articles and conference proceedings and contacted the authors of included trials.

The search strategy was: (tDCS OR TDCS OR Cathodal Stimulation Transcranial Direct Current Stimulation OR Cathodal Stimulation tDCSs OR Cathodal Stimulation tDCS OR Transcranial Random Noise Stimulation OR Transcranial Alternating Current Stimulation OR Transcranial Electrical Stimulation OR dual transcranial direct current stimulation OR Transcranial Electrical Stimulations OR Anodal Stimulation Transcranial Direct Current Stimulation OR Anodal Stimulation Tdcs OR Anodal Tdcs OR Anodal Stimulation TDCSs OR Repetitive Transcranial Electrical Stimulation OR repetitive transcranial magnetic stimulation OR RTMS OR rTMS OR High-frequency rTMS OR Trasncranial Magnetic Stimulation OR Transcranial Magnetic Stimulations OR Low-frequency transcranial magnetic stimulation OR Stimulation Transcranial Magnetic OR Stimulations Transcranial Magnetic OR Single Pulse Transcranial Magnetic Stimulation OR Paired Pulse Transcranial Magnetic Stimulation OR Repetitive Transcranial Magnetic Stimulation OR theta burst OR theta burst stimulation OR theta-burst OR theta-burst stimulation OR burst stimulation OR continuous theta burst stimulation OR continuous
TBS OR TBS) AND (cerebrovascular disorders OR basal ganglia cerebrovascular disease OR hemispatial neglect OR hemispatial neglect OR spatial attentional asymmetries OR brain ischemia OR carotid artery diseases OR intracranial arterial diseases OR intracranial embolism and thrombosis OR intracranial hemorrhages OR stroke OR brain infarction OR vertebral artery dissection OR post-stroke OR poststroke OR hemineglect OR hemi-neglect OR unilateral visuospatial neglect OR visuospatial neglect OR visual spatial neglect OR spatial neglect OR unilateral neglect of acute stroke patients OR unilateral spatial neglect OR patients with stroke OR stroke patients with spatial neglect $\mathrm{OR}$ right hemisphere strokes OR rehabilitation after stroke OR chronic spatial neglect after stroke OR unilateral neglect OR spatial neglect OR hemispatial neglect OR visual neglect OR inattention OR hemi-inattention OR space perception OR visual perception OR perceptual disorders OR perceptual disorder OR extinction $\mathrm{OR}$ functional laterality).

2.3. Selection of Studies. Two pairs of reviewers independently screened all titles and abstracts identified by the literature search, obtained full-text articles of all potentially eligible studies, and evaluated them for eligibility. Reviewers resolved disagreement by discussion or, if necessary, with third party adjudication. We also considered studies reported only as conference abstracts.

2.4. Data Extraction. Reviewers underwent calibration exercises and worked in pairs to independently extract data from included studies. They resolved disagreement by discussion or, if necessary, with third party adjudication. They abstracted the following data using a pretested data extraction form: study design, participants, interventions, comparators, outcome assessed, and relevant statistical data.

2.5. Risk of Bias Assessment. Reviewers, working in pairs, independently assessed the risk of bias of included RCTs using a modified version of the Cochrane Collaboration's instrument (http:/distillercer.com/resources/) [39]. That version includes nine domains: adequacy of sequence generation, allocation sequence concealment, blinding of participants and caregivers, blinding of data collectors, blinding for outcome assessment, blinding of data analysts, incomplete outcome data, selective outcome reporting, and the presence of other potential sources of bias not accounted for in the previously cited domains [40]. For incomplete outcome data in individual studies, we stipulated as low risk of bias for loss to follow-up as less than $10 \%$ and a difference of less than $5 \%$ in missing data between intervention/exposure and control groups.

When information regarding risk of bias or other aspects of methods or results was unavailable, we attempted to contact study authors for additional information.

2.6. Certainty of Evidence. We summarized the evidence and assessed its certainty separately for bodies of evidence from RCT and non-RCT studies. We used the Grading of Recommendations Assessment, Development and Evaluation (GRADE) methodology to rate certainty of the evidence for 
each outcome as high, moderate, low, or very low [41]. In the GRADE approach, RCTs begin as high certainty and nonRCT studies begin as moderate certainty. Detailed GRADE guidance was used to assess overall risk of bias [42], imprecision [43], inconsistency [44], indirectness [45], and publication bias [46] and to summarize the results in an evidence profile (Table 1).

We planned to assess publication bias through visual inspection of funnel plots for each outcome in which we identified 10 or more eligible studies; however, we were not able to do so because there were an insufficient number of studies to allow for this assessment.

2.7. Data Synthesis and Statistical Analysis. We calculated pooled inverse variance standardized mean difference (SMD) and associated 95\% CIs using random-effects models. We addressed variability in results across studies by using $I^{2}$ statistic and the $P$ value obtained from the Cochran chi square test. Our primary analyses were based on eligible patients who had reported outcomes for each study (complete case analysis). We used Review Manager (RevMan) (version 5.3; Nordic Cochrane Centre, Cochrane) for all analyses [47].

2.8. Subgroup and Sensitivity Analyses. We planned possible subgroup analyses according to the following characteristics:

(i) Participants (stroke type: ischemic stroke versus intracranial hemorrhage)

(ii) Interventions (type of stimulation: cathodal versus anodal and position of electrodes; type of frequency: high frequency versus low frequency)

(iii) Comparator (type of control intervention: pharmacological therapy versus nonpharmacological therapy)

(iv) Different tests for overall USN (star cancellation test versus line bisection test)

We planned to conduct subgroup analyses only when five or more studies were available, with at least two in each subgroup. We planned to synthesize the evidence separately for bodies of evidence from RCT and non-RCT studies by a sensitivity analysis.

\section{Results}

3.1. Study Selection. We identified a total of 4129 citations through database searches and a further four studies from the reference lists of the Cochrane reviews [22, 48, 49] (see Figure 1 for search results). After screening by title and then by abstract, we obtained full-paper copies for 30 citations that were potentially eligible for inclusion in the review. We excluded 15 studies for the following reasons: case report, case series, self-controlled study, review, and off-topic. The remaining 12 RCTs $[14,50-60]$ with a total of 273 participants and four non-RCTs [61-64] with a total of 94 participants met the minimum requirements, and we included them in this review.
3.2. Study Characteristics. Table 2 describes study characteristics related to design of study, setting, number of participants, mean age, gender, inclusion and exclusion criteria, and follow-up. Eight studies [14, 54, 56, 59, 61-64] were conducted largely in Europe and eight in Asia [50-53, 55, 57, 58, 60]. Randomized trials' sample sizes ranged from 10 [56] to 38 [55], and non-RCT studies ranged from 12 [63] to 36 [62]. Typical participants were males in their 40s, 50s, and 60s. Studies followed participants immediately after treatment $[50,57,58,62]$ to one month $[51,52,54-56]$.

Table 3 describes study characteristics related to intervention and comparators and assessed outcomes. Of the 16 included studies, nine trials $[14,50,52,54,55,59-62]$ evaluated TBS:

(1) Eight trials compared cTBS versus

(i) sham cTBS (both groups received conventional rehabilitation training $[52,60])$;

(ii) $1 \mathrm{~Hz}$ rTMS, $10 \mathrm{~Hz}$ rTMS, and sham rTMS (all groups with addition of routine rehabilitation [55]);

(iii) sham TBS [14];

(iv) sham cTBS $[54,59,61,62]$.

(2) One trial compared iTBS with $80 \%$ resting motor threshold (RMT) versus iTBS 40\% RMT [50].

Of the remaining seven studies, four trials $[56-58,64]$ evaluated tDCS:

(1) Two trials compared tDCS over the left (cathodal) and right (anodal) posterior parietal cortex, one versus placebo at an intensity of $2 \mathrm{~mA}$ [56] and the other versus sham tDCS [64].

(2) One trial [57] compared tDCS dual versus either tDCS single or tDCS sham.

(3) One trial [58] compared tDCS versus sham tDCS.

Three further trials $[51,53,63]$ evaluated rTMS:

(1) One trial [51] compared rTMS with sham rTMS, both plus conventional rehabilitation therapy (neurodevelopmental facilitation techniques).

(2) Two trials compared $1 \mathrm{~Hz}$ rTMS, one versus $10 \mathrm{~Hz}$ rTMS and sham rTMS [53] (both groups received conventional rehabilitation), and the other trial compared $1 \mathrm{~Hz}$ rTMS versus sham rTMS [63].

None of the included studies evaluated noninvasive brain stimulations as an adjunct to any type of conventional stroke rehabilitation.

3.3. Risk of Bias. Figure 2 describes the risk of bias assessment for the RCTs and non-RCTs, respectively. The major issue 


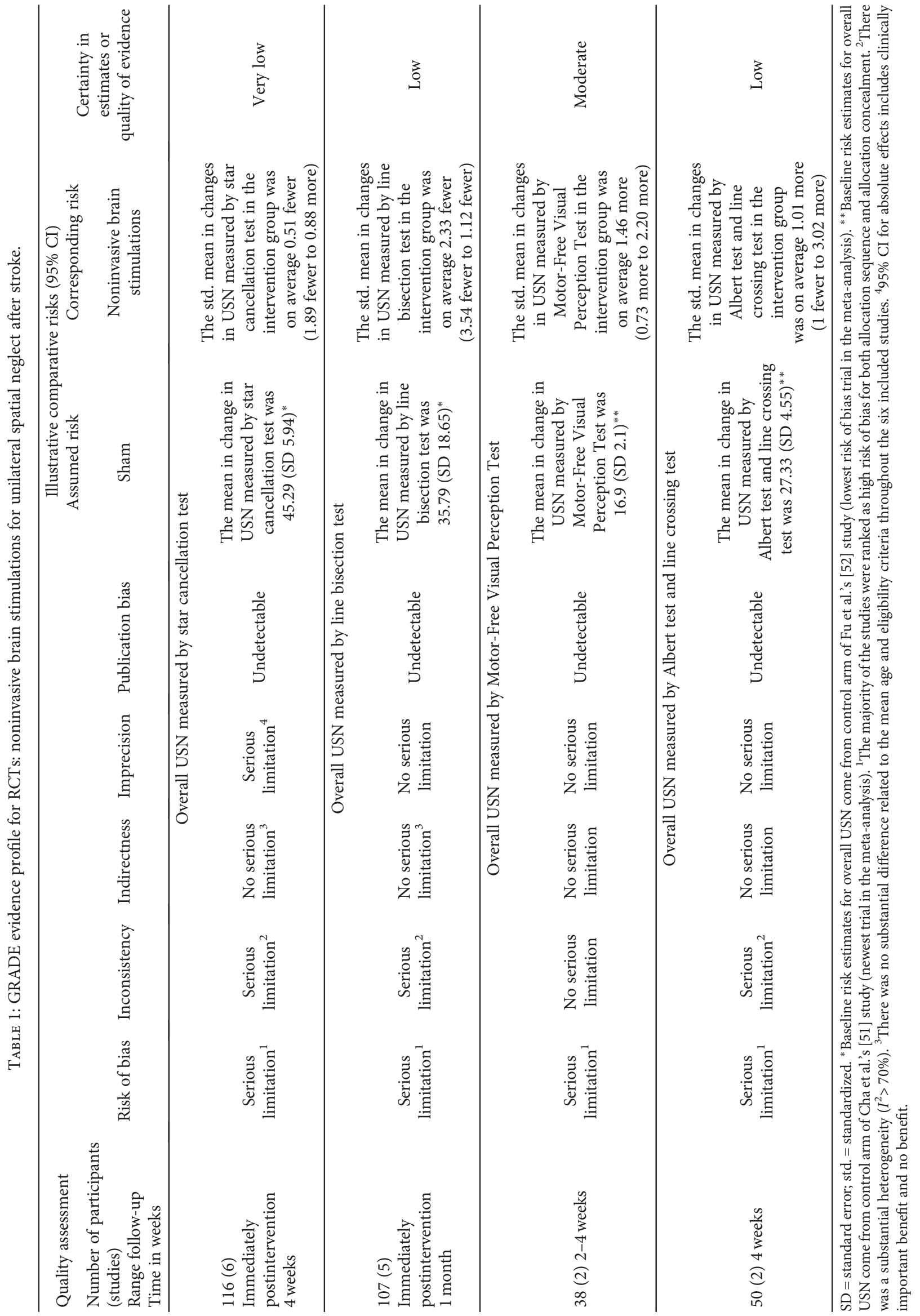




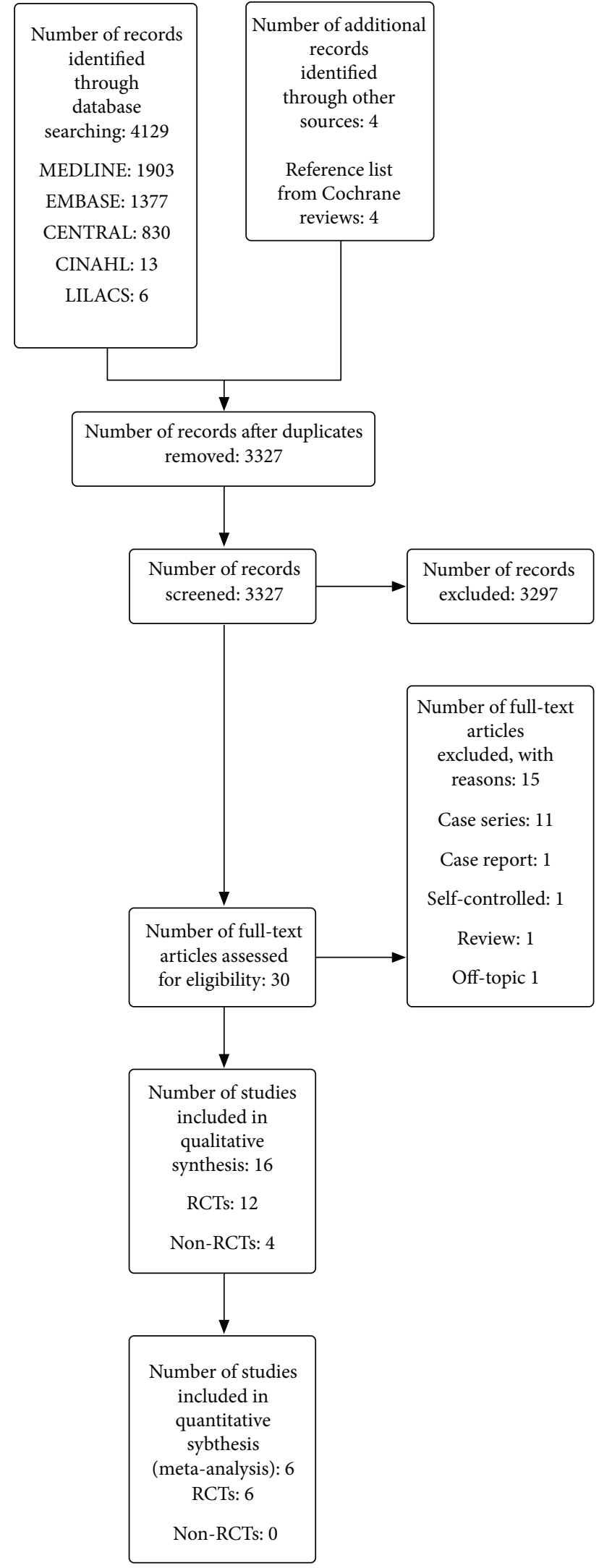

FIGURE 1: Flow diagram of the systematic review. regarding risk of bias in the RCTs and non-RCTs was problems of random sequence generation $[14,50,51,54-59,61-64]$ and concealment of randomization [14, 50, 53-59, 61-64]. An additional problem was blinding of the statistician in all included studies.

\subsection{Outcomes}

\subsubsection{Synthesized Results from Randomized Controlled Trials}

(1) Overall USN Measured by the Star Cancellation Test. The results from six RCTs [51-55, 57] comparing noninvasive brain stimulations with sham failed to show a benefit in overall USN measured by the star cancellation test (SMD -0.51, 95\% CI $-1.87,0.85 ; p=0.46 ; I^{2}=90 \%$ ) (Figure 3). The results were consistent regardless of the type of noninvasive brain stimulations (TBS in three RCTs [52, 54, 55] (SMD $-1.61,95 \%$ CI $\left.-4.28,1.06 ; p=0.24 ; I^{2}=93 \%\right)$; dual-tDCS in one RCT [57] (SMD $-0.12,95 \%$ CI $-0.99,0.76 ; p=0.79$; $I^{2}=$ not applicable); and $1 \mathrm{~Hz}$ rTMS in two RCTs $[51,53]$ (SMD 0.57, 95\% CI $\left.-2.95,4.10 ; p=0.75 ; I^{2}=95 \%\right)$ ) (Figure 3). Certainty in evidence was rated as very low because of imprecision, inconsistency, and risk of bias due to the studies that were ranked as high risk of bias for both allocation sequence and allocation concealment (Figure 2).

A sensitivity analysis from the same RCTs using TBS [52, 54, 55], single-tDCS [57], and $10 \mathrm{~Hz}$ rTMS [51, 53] yielded results that were also consistent with the primary analysis and failed to show a difference in the effects of noninvasive brain stimulations compared to sham (SMD $-0.62,95 \% \mathrm{CI}-1.89,0.65 ; p=0.34 ; I^{2}=88 \%$ ) (Figure 4 ).

(2) Overall USN Measured by the Line Bisection Test. Results from five RCTs [51-53, 55, 57] comparing noninvasive brain stimulations with sham suggested a benefit in overall USN measured by the line bisection test (SMD $-2.33,95 \%$ CI $-3.54,-1.12 ; p=0.0002 ; I^{2}=81 \%$ ) (Figure 5). The results were inconsistent when the data were analyzed by type of noninvasive brain stimulations: TBS in two RCTs $[52,55]$ (SMD -3.08, 95\% CI -6.54, 0.38; $\left.p=0.08 ; I^{2}=90 \%\right)$ and dual-tDCS in one RCT [57] (SMD $-0.66,95 \%$ CI -1.56 , $0.25 ; p=0.15 ; I^{2}=$ not applicable) except by $1 \mathrm{~Hz}$ rTMS in two RCTs $[51,53]$ that yielded results that were consistent with the overall meta-analysis (SMD $-2.33,95 \%$ CI $-3.54,-1.12 ; p<0.0002 ; I^{2}=81 \%$ ) (Figure 5). Certainty in evidence was rated as low because of inconsistency and risk of bias due to the studies that were ranked as high risk of bias for both allocation sequence and allocation concealment (Figure 2).

A sensitivity analysis from the same RCTs using TBS [52, 55], tDCS [57], and $10 \mathrm{~Hz}$ rTMS [53] yielded results that were also consistent with the primary analysis and suggested a difference in the effects of noninvasive brain stimulations compared to sham (SMD $-2.35,95 \% \mathrm{CI}-3.72,-0.98$; $\left.p=0.002 ; I^{2}=85 \%\right)($ Figure 6$)$.

(3) Overall USN Measured by Motor-Free Visual Perception Test. The results from two RCTs $[51,53]$ comparing noninvasive brain stimulations with sham suggested a benefit 


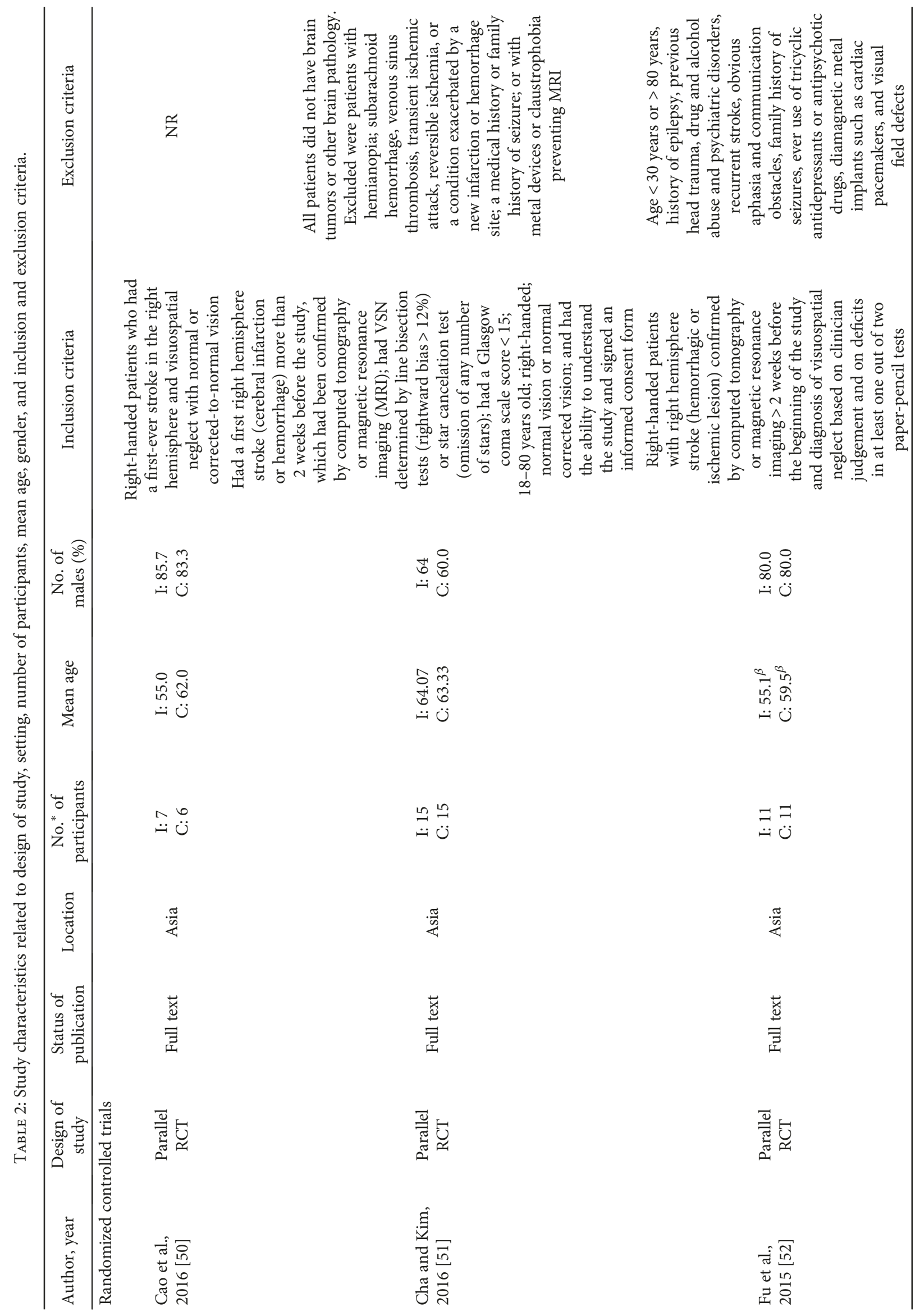




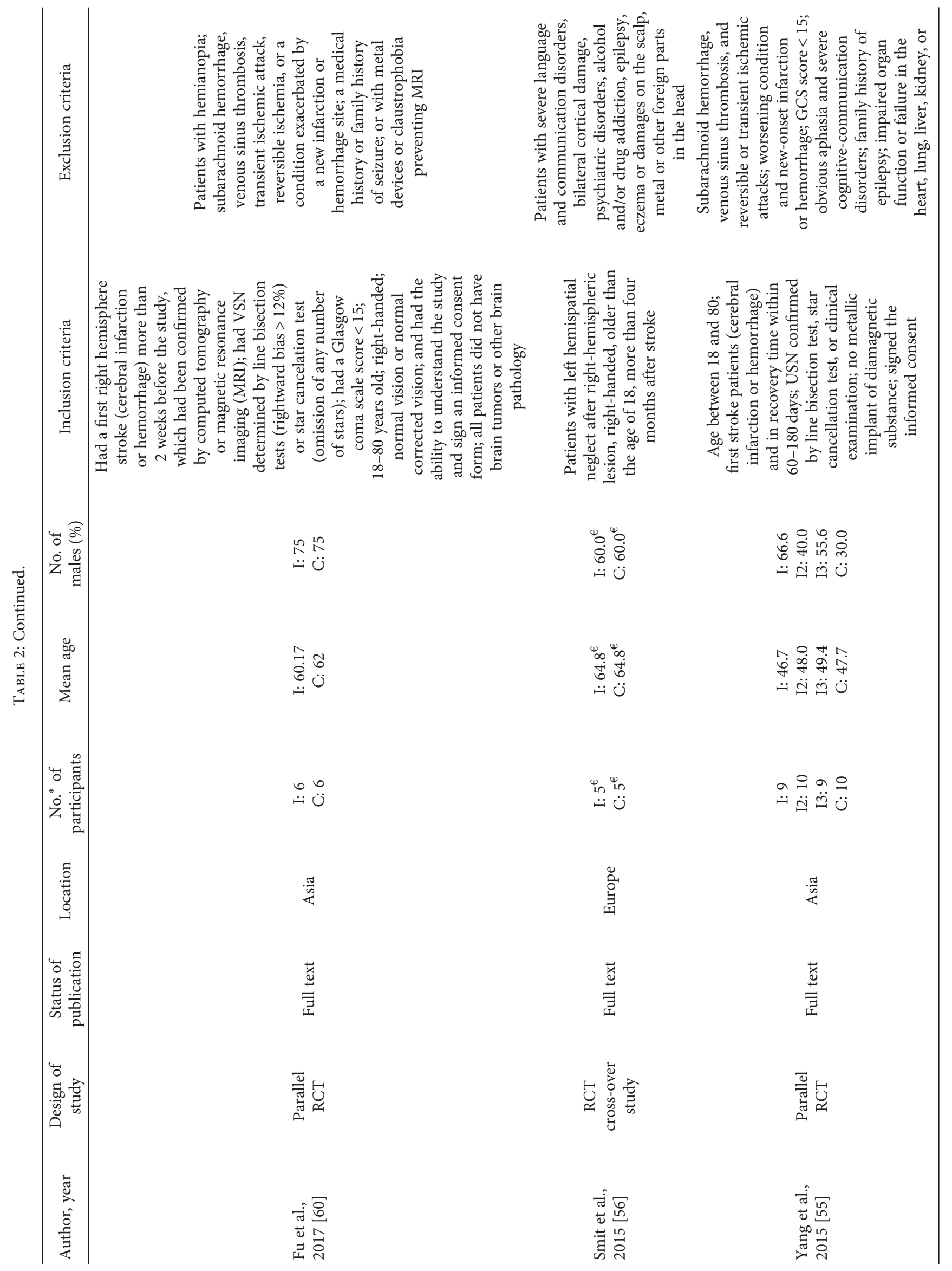




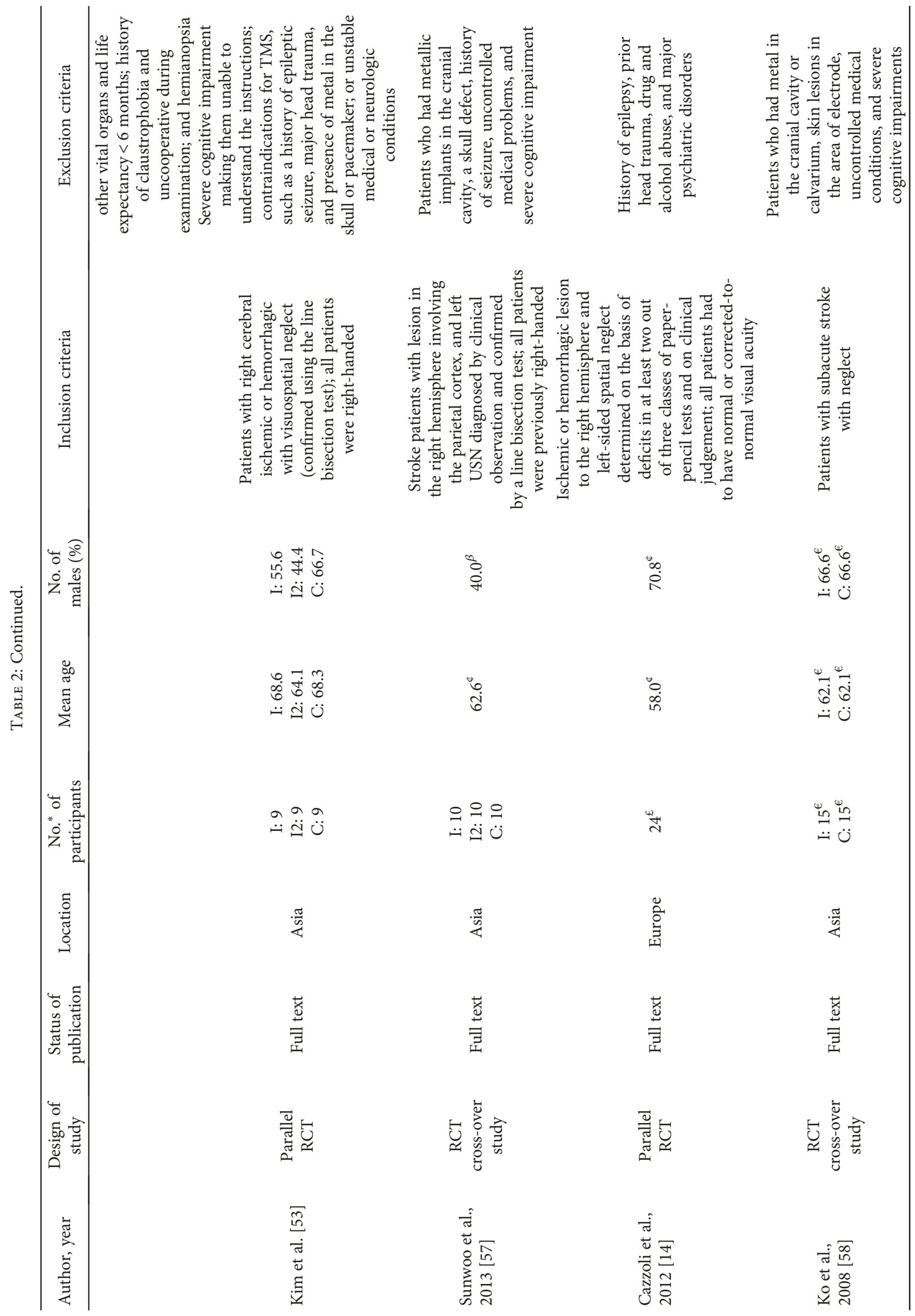




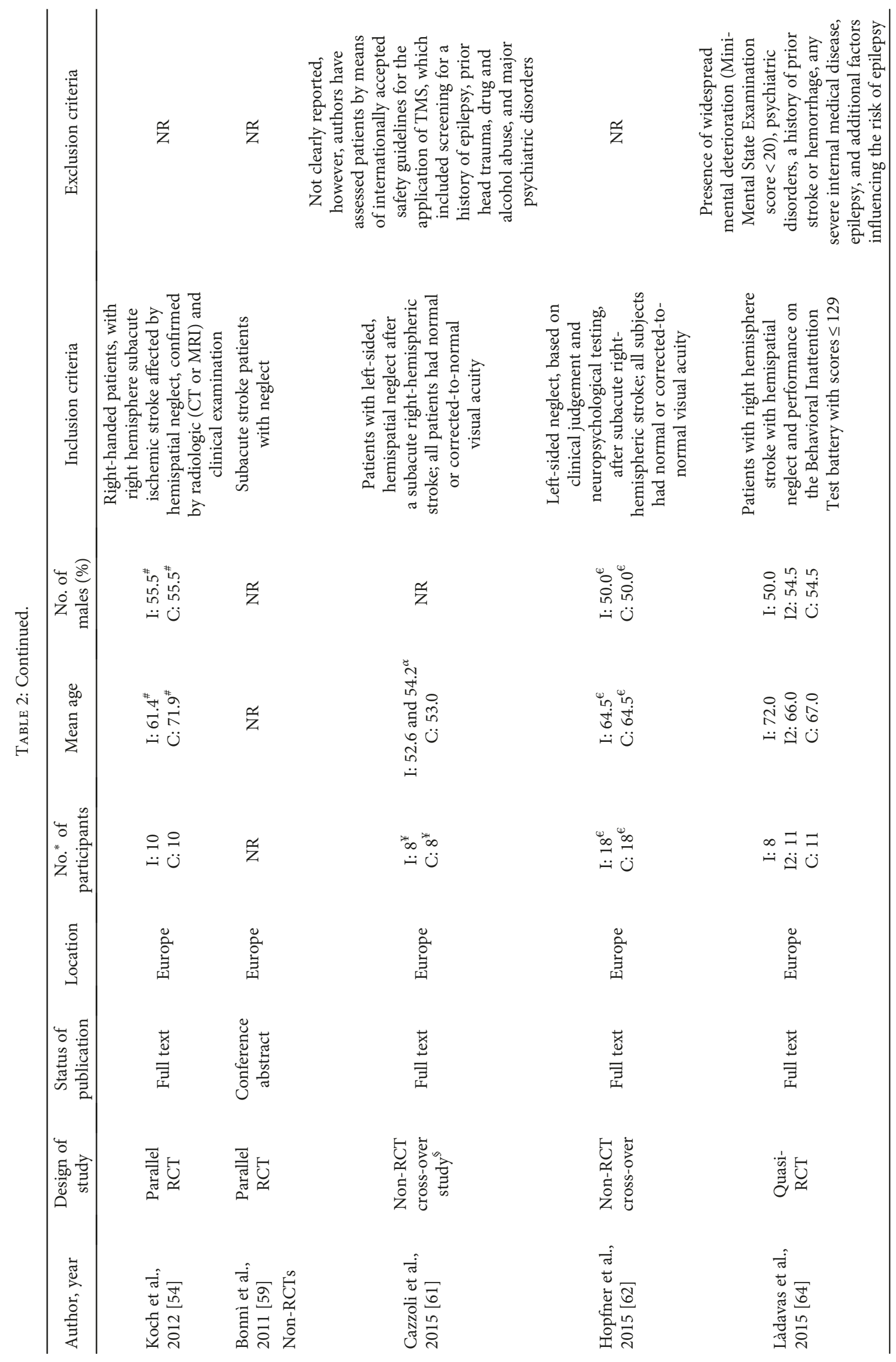




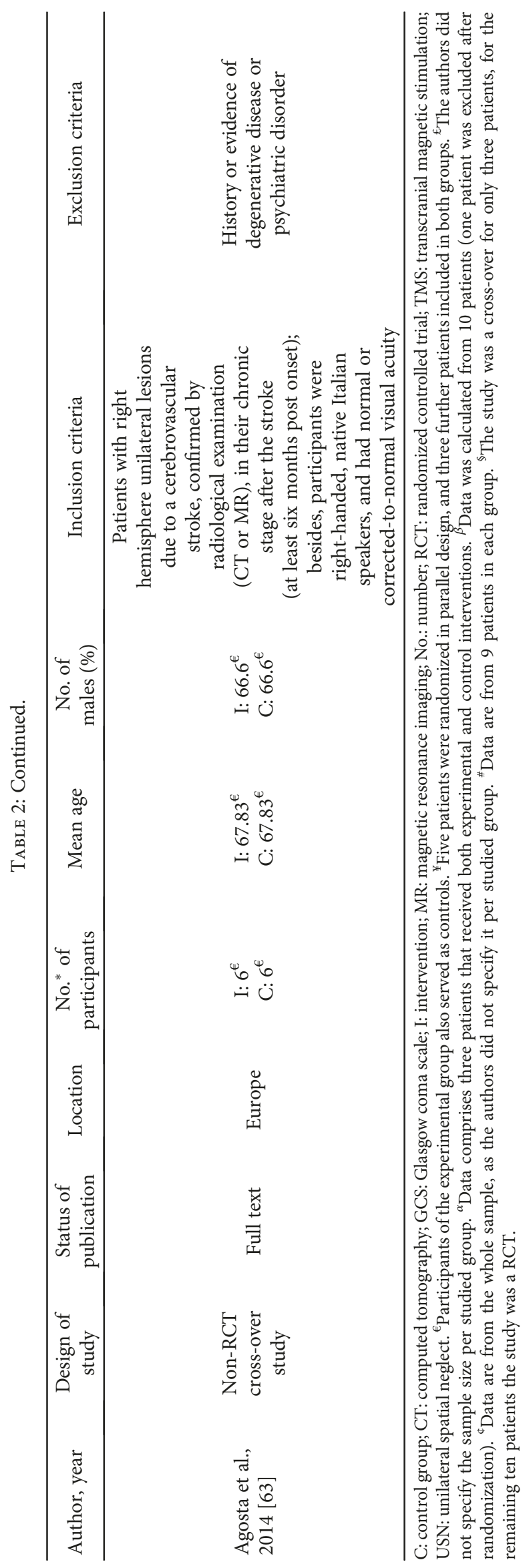




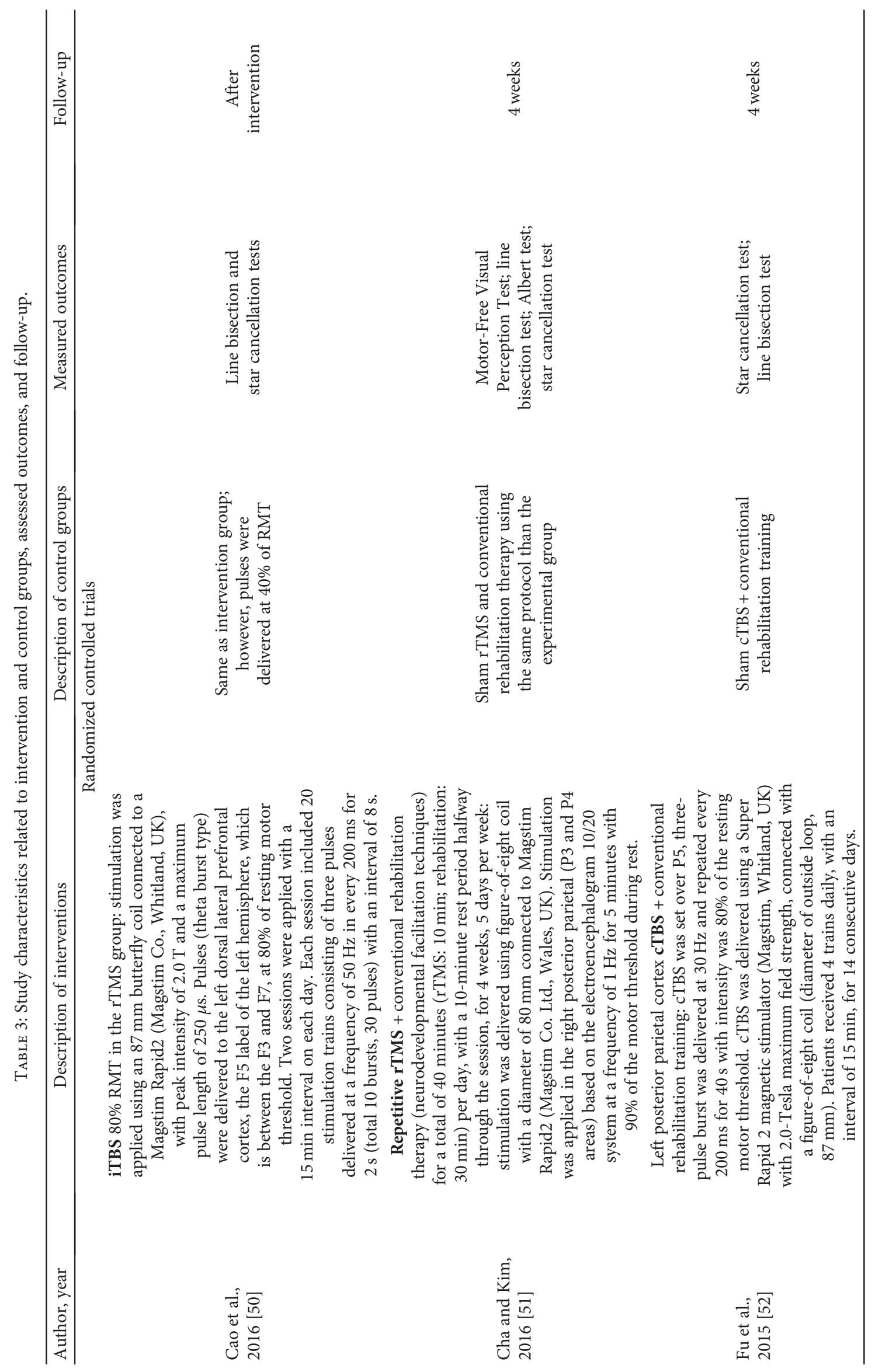




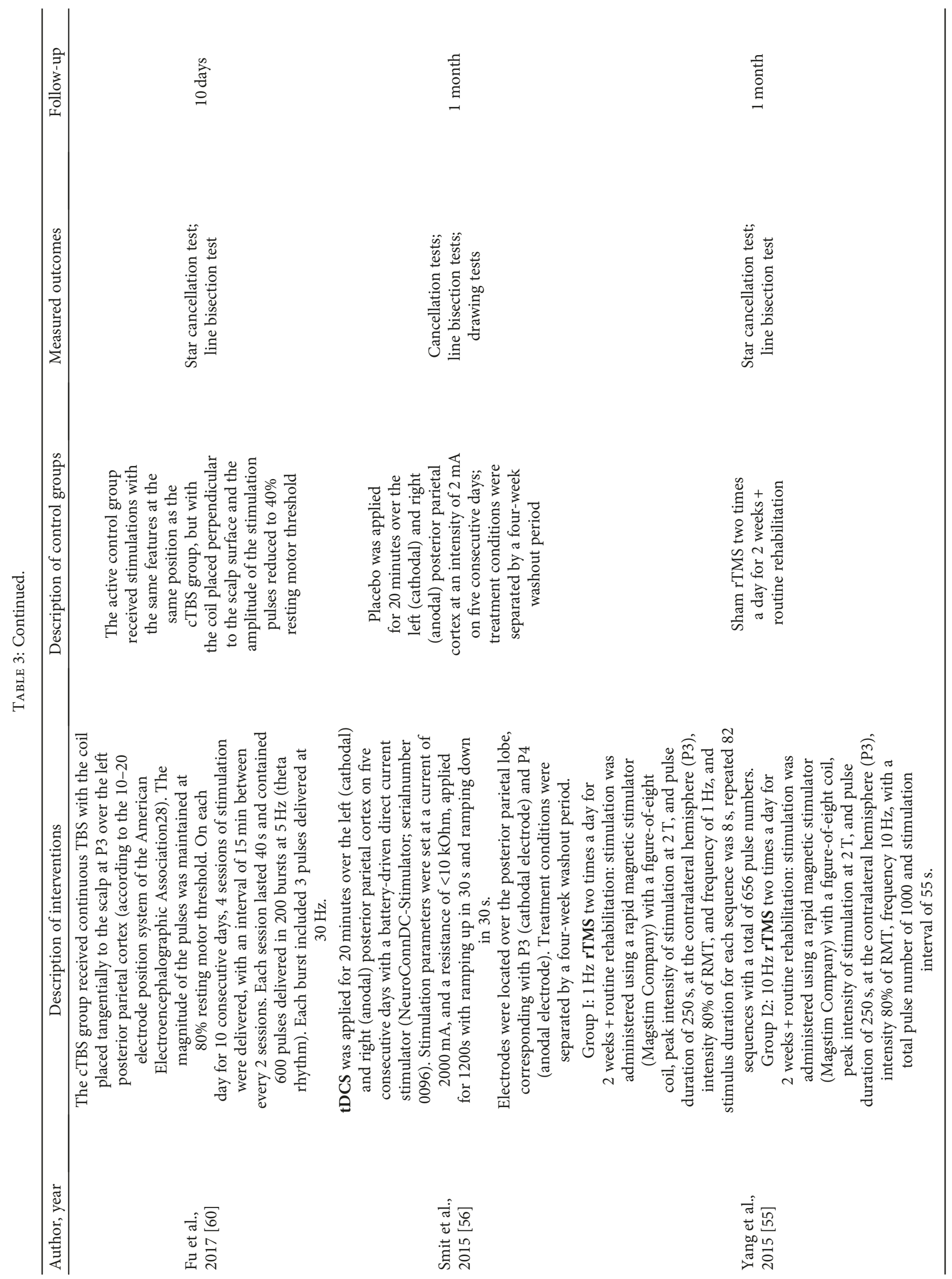




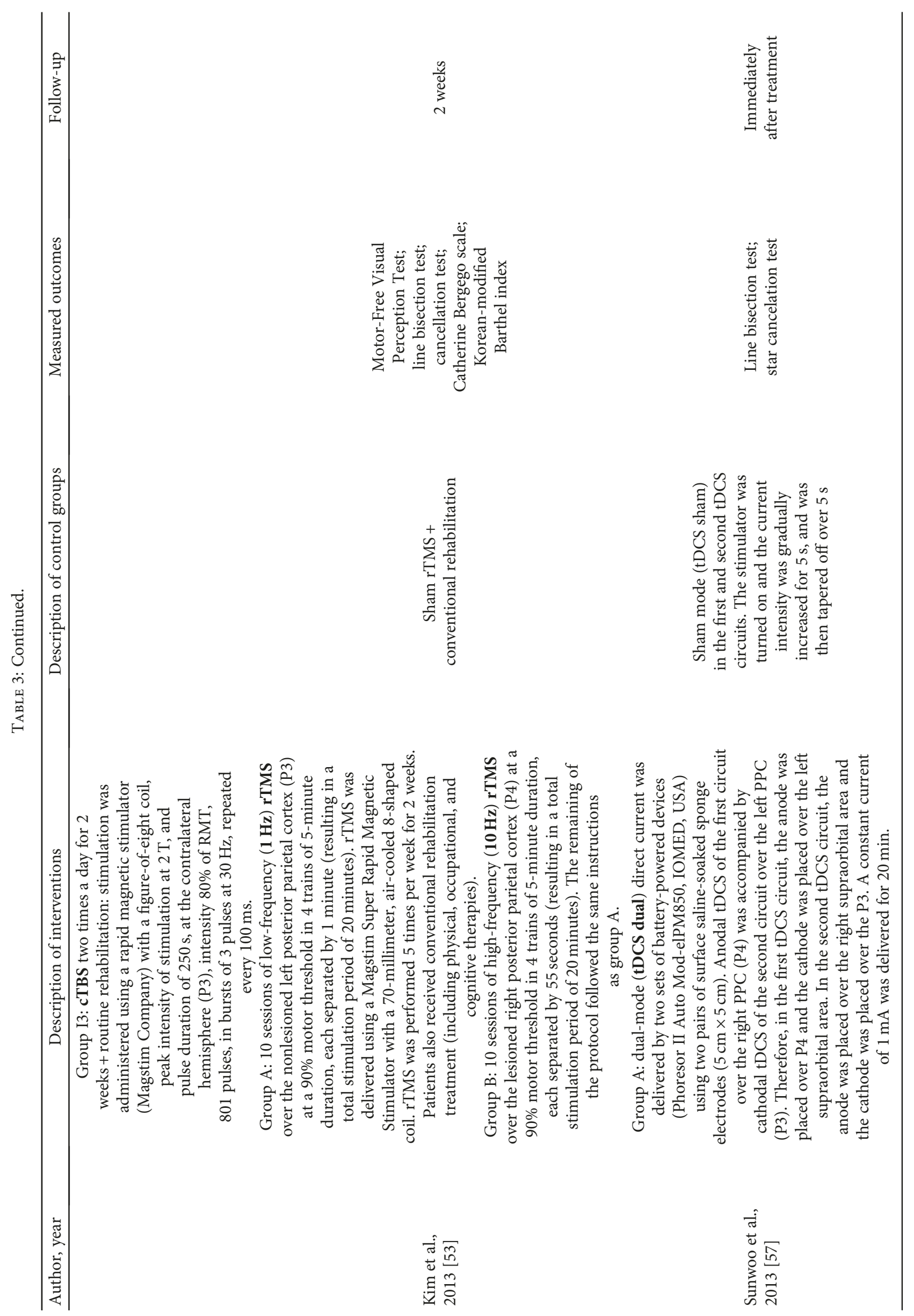



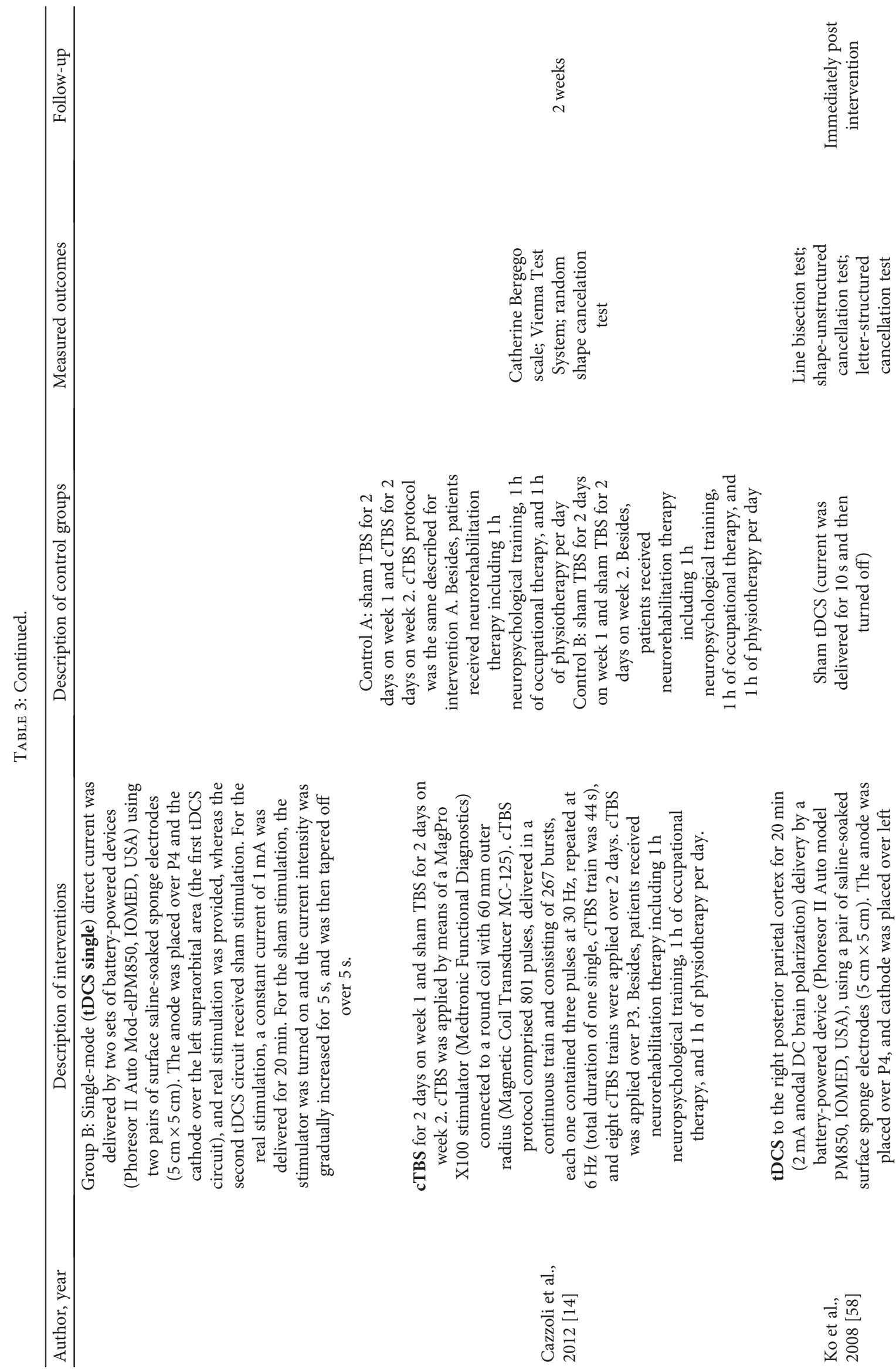

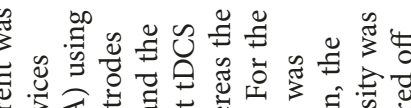

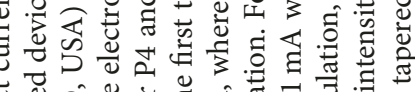
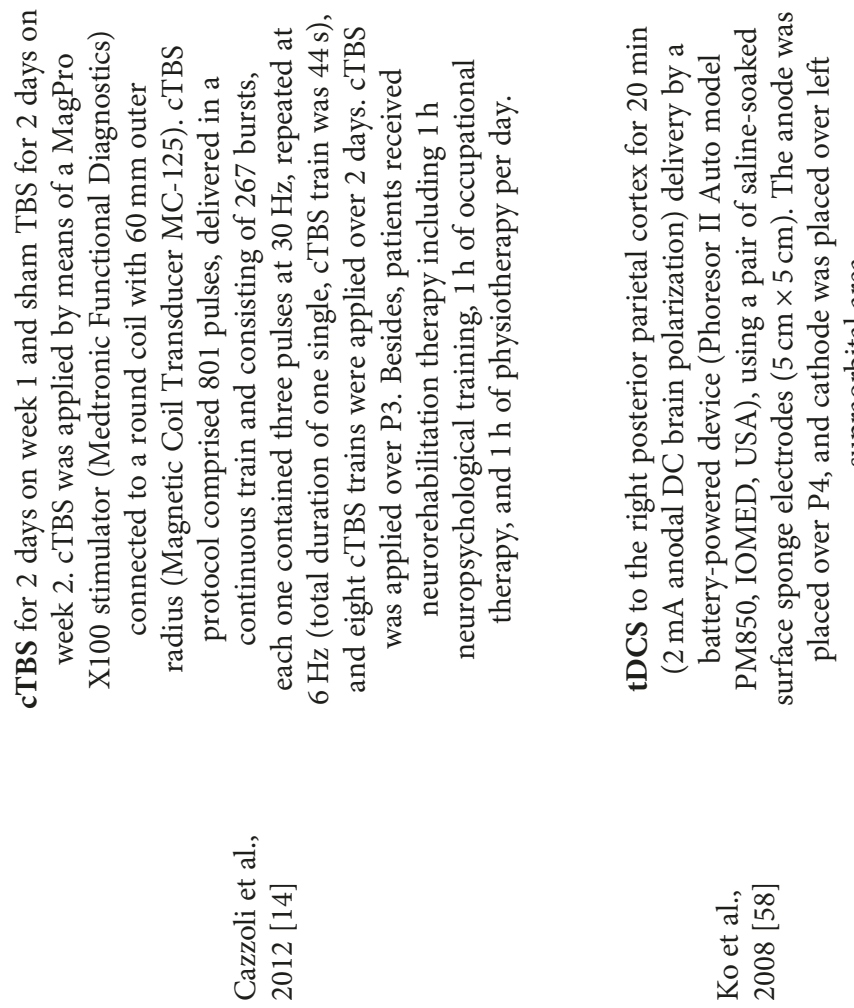

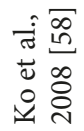




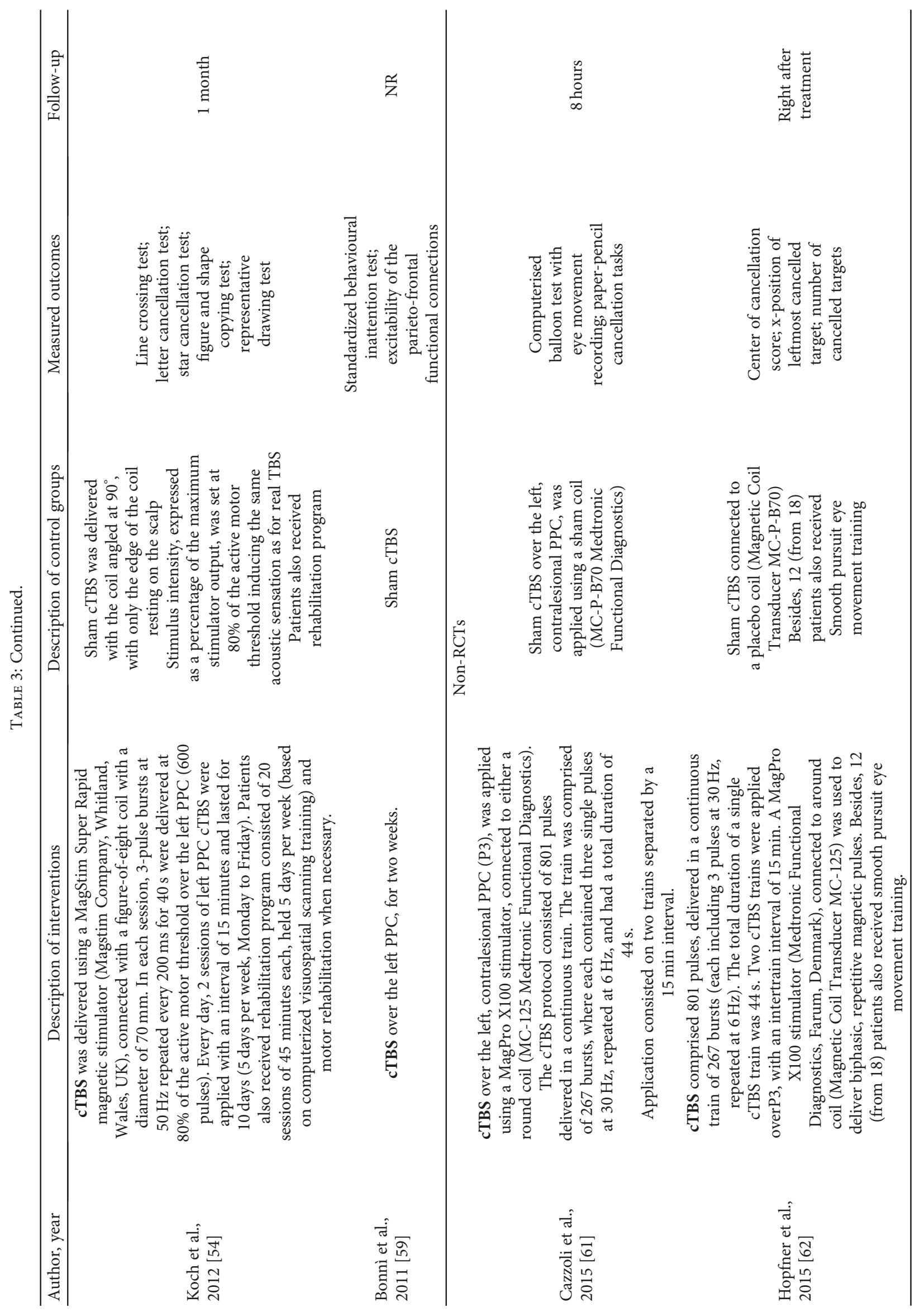




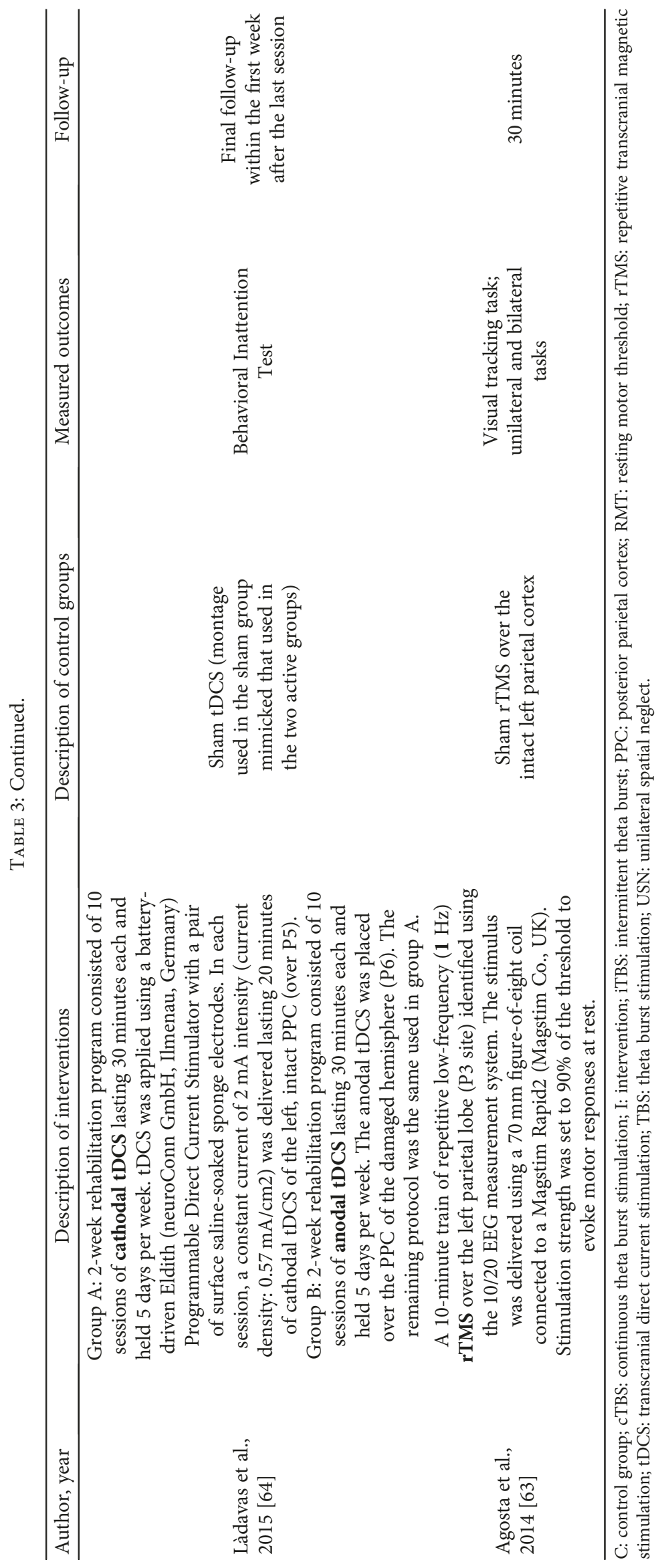




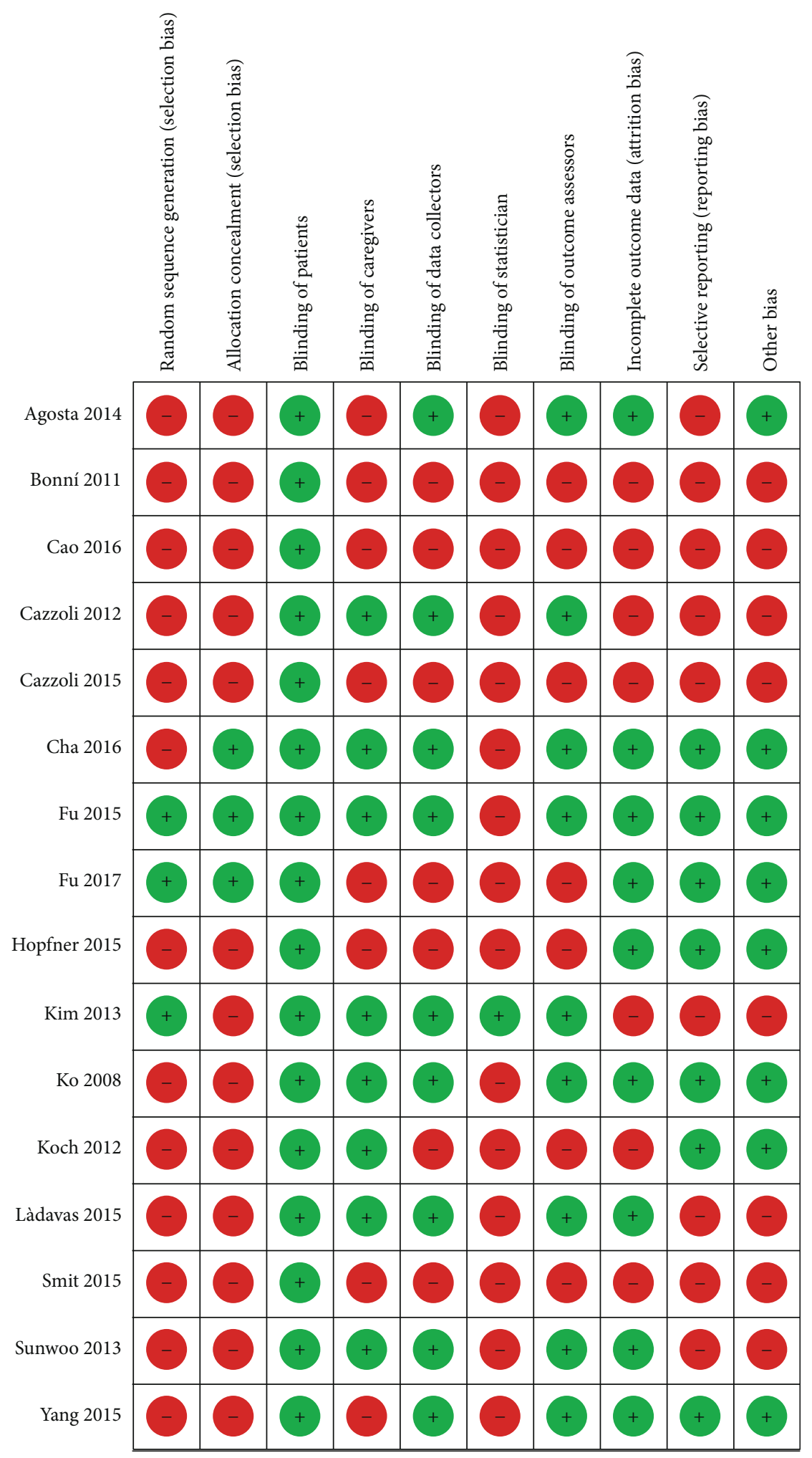

FIGURE 2: Risk of bias assessment for RCTs and non-RCTs.

in overall USN measured by the Motor-Free Visual Perception Test at $1 \mathrm{~Hz}\left(\mathrm{SMD} 1.46,95 \% \mathrm{CI} 0.73,2.20 ; p<0.0001 ; I^{2}=0 \%\right.$ ), and the difference was not observed using $10 \mathrm{~Hz}$ (SMD 0.97, 95\% CI $-0.02,1.96 ; p=0.06 ; I^{2}=$ not applicable) (Figure 7).
Certainty in evidence was rated as moderate because of risk of bias due to the studies that were ranked as high risk of bias for both allocation sequence and allocation concealment (Figure 2). 


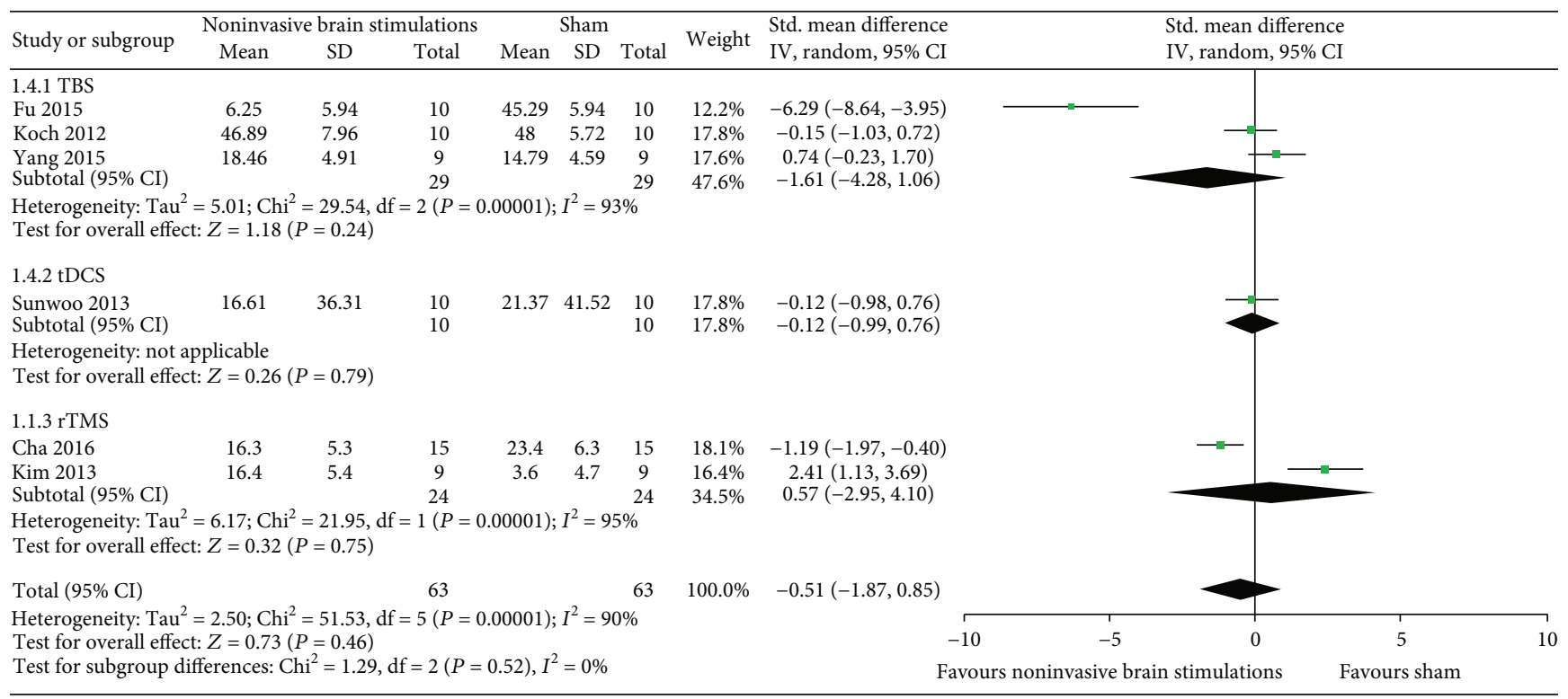

Figure 3: Meta-analysis of overall USN measured by the star cancellation test.

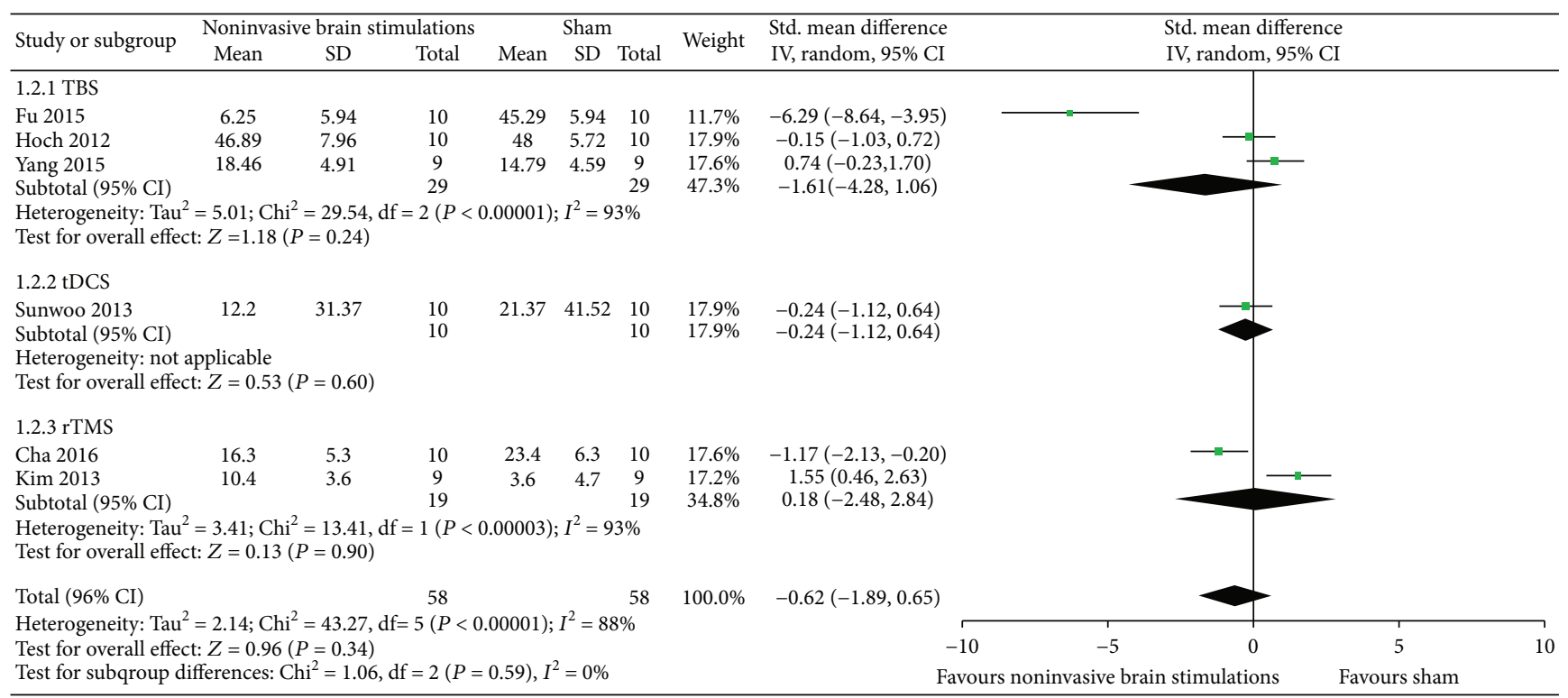

FIGURE 4: Sensitivity analysis of overall USN measured by the star cancellation test using TBS, single-mode tDCS, and $10 \mathrm{~Hz}$ rTMS.

(4) Overall USN Measured by Albert's Test and the Line Crossing Test. The results from two RCTs [51, 54] comparing noninvasive brain stimulations with sham failed to show a benefit in overall USN measured by Albert's test and the line crossing test (SMD 1.01, 95\% CI -1.0, 3.02; $p=0.32 ; I^{2}=90.2 \%$ ) (Figure 8). However, in the subgroup analysis with the use of $1 \mathrm{~Hz}$ rTMS, we found a statistically significant difference compared to sham (SMD 2.04, $95 \%$ CI $1.14,2.95 ; p<0.00001 ; I^{2}=$ not applicable). Regarding the use of TBS, there was no benefit compared to sham (SMD $-0.01,95 \%$ CI $-0.89,0.87 ; p=0.98 ; I^{2}=$ not applicable). Certainty in evidence was rated as low because of inconsistency and risk of bias due to the studies that were ranked as high risk of bias for both allocation sequence and allocation concealment (Figure 2).
(5) Other Outcomes: Daily Life Functions and Adverse Events. Only Kim et al. [53] reported on daily life functions with a higher mean in the $10 \mathrm{~Hz}$ rTMS group than in the sham and $1 \mathrm{~Hz}$ rMTS groups; however, there was only a statistically significant difference favoring the $10 \mathrm{~Hz}$ rTMS group compared to the sham group (SMD 1.83, 95\% CI 0.68, 2.97; $p=0.002 ; I^{2}=$ not applicable). Làdavas et al.'s study [64] was the only study that reported on adverse events; no significant adverse effect of tDCS was reported, except only a few cases of minimal irritation of the skin beneath the electrodes.

None of the included studies reported on the following outcomes: neurological and functional disabilities, loss of balance, depression or anxiety and evaluation of poststroke fatigue, quality of life, and death. 


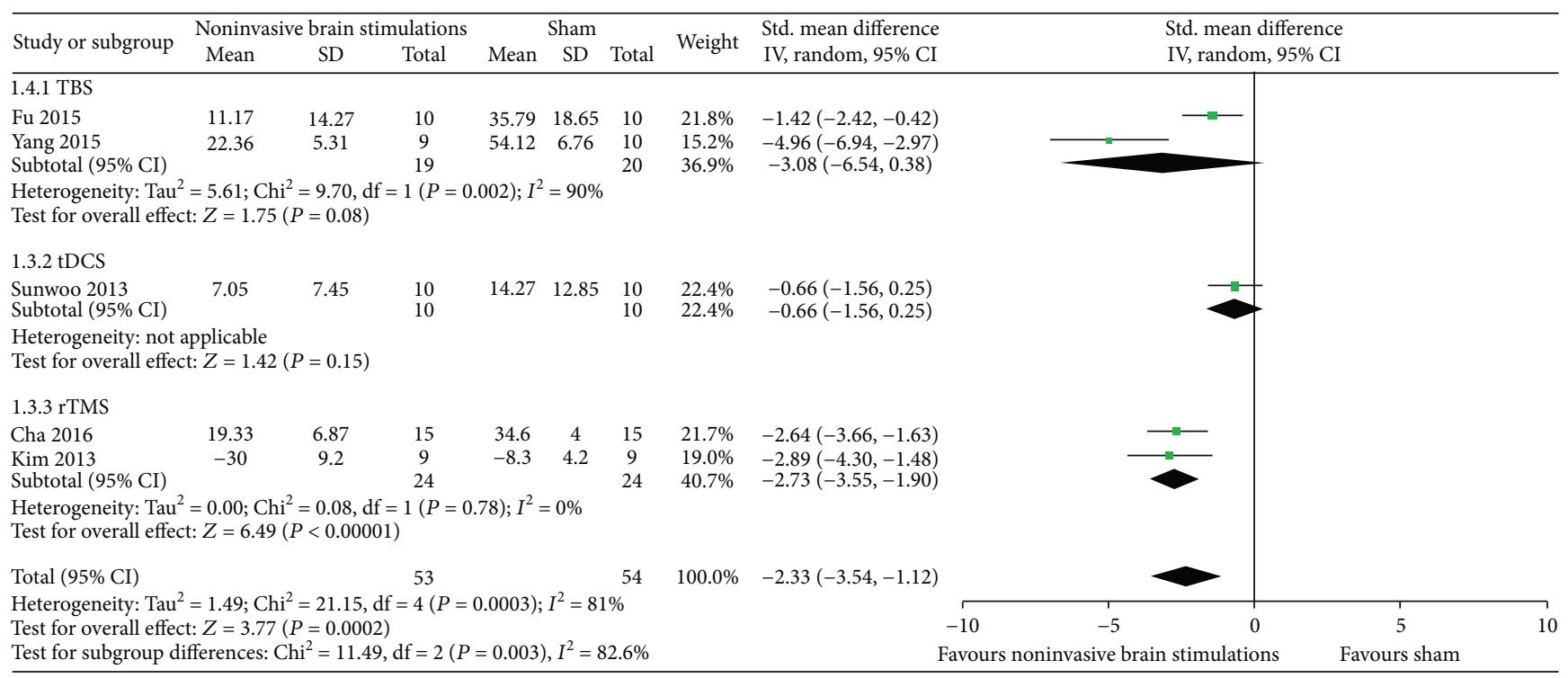

FIGURE 5: Meta-analysis of overall USN measured by the line bisection test.

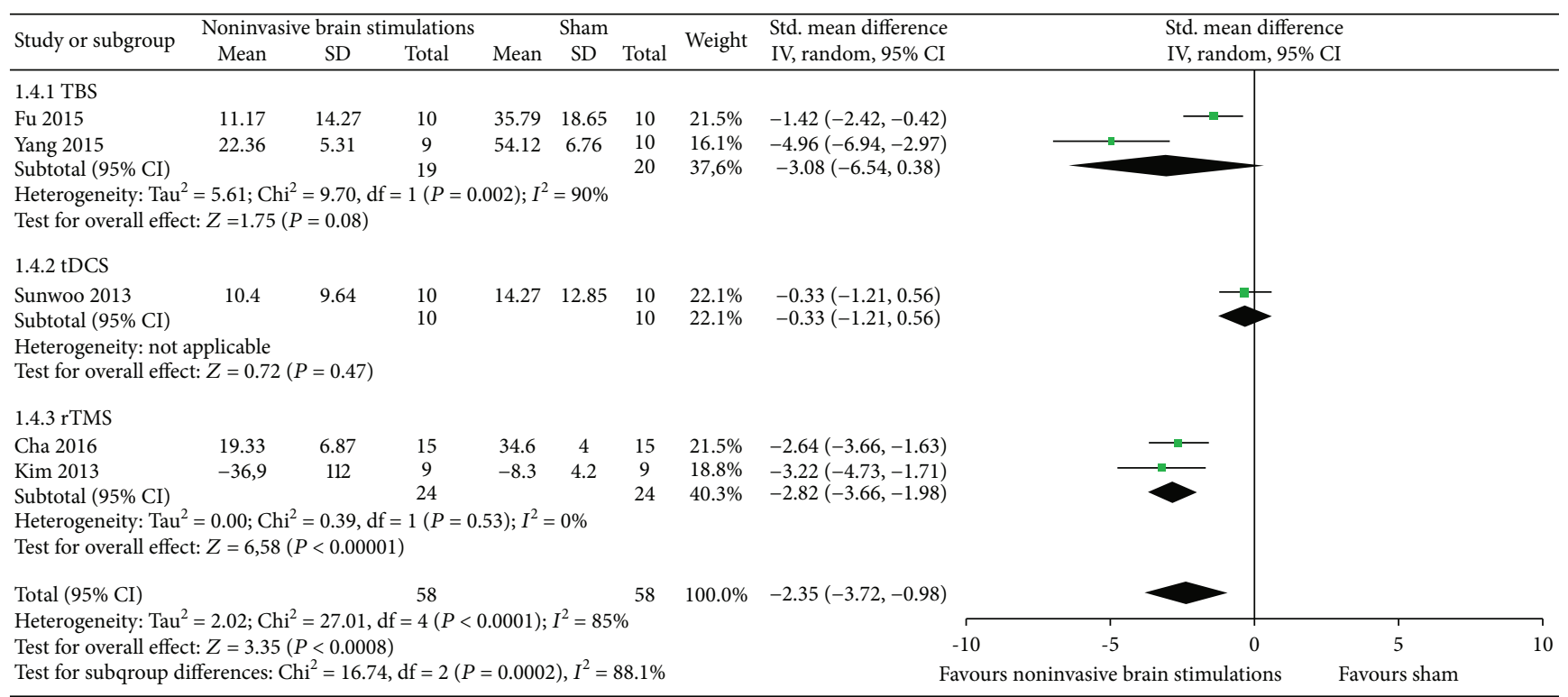

FIGURE 6: Sensitivity analysis of overall USN measured by the line bisection test using TBS, single-mode tDCS, and $10 \mathrm{~Hz}$ rTMS.

3.4.2. Synthesized Results from Non-RCTs. The non-RCTs did not report data in a usable way to allow for any statistical analysis.

\section{Discussion}

4.1. Main Findings. Based on pooled data from six randomized trials with 116 participants, we found evidence for a benefit in overall USN with noninvasive brain stimulation, especially with the use of rTMS in comparison to the sham (Figures 5, 7, and 8). The evidence is from moderatequality evidence because of risk of bias due to the studies that were ranked as high risk of bias for both allocation sequence and allocation concealment (Figure 2). Non-RCT studies provided no evidence, suggesting that future trials should adhere to CONSORT guidelines to ensure clarity and reproducibility in the reporting of methods.

We presented the results of overall USN in a forest plot, which showed a statistically significant difference between the noninvasive brain stimulations and sham in the following tests: line bisection test, Motor-Free Visual Perception Test, and Albert's test and line crossing test. Nevertheless, the study also showed a nonsignificant difference between the noninvasive brain stimulations and sham on the star cancellation test.

Several noninvasive brain stimulations have been explored to determine whether some of these techniques might be useful in promoting recovery from USN after stroke. The lesion of the right parietal cortex after stroke causes disinhibition of the left hemisphere and thus a 


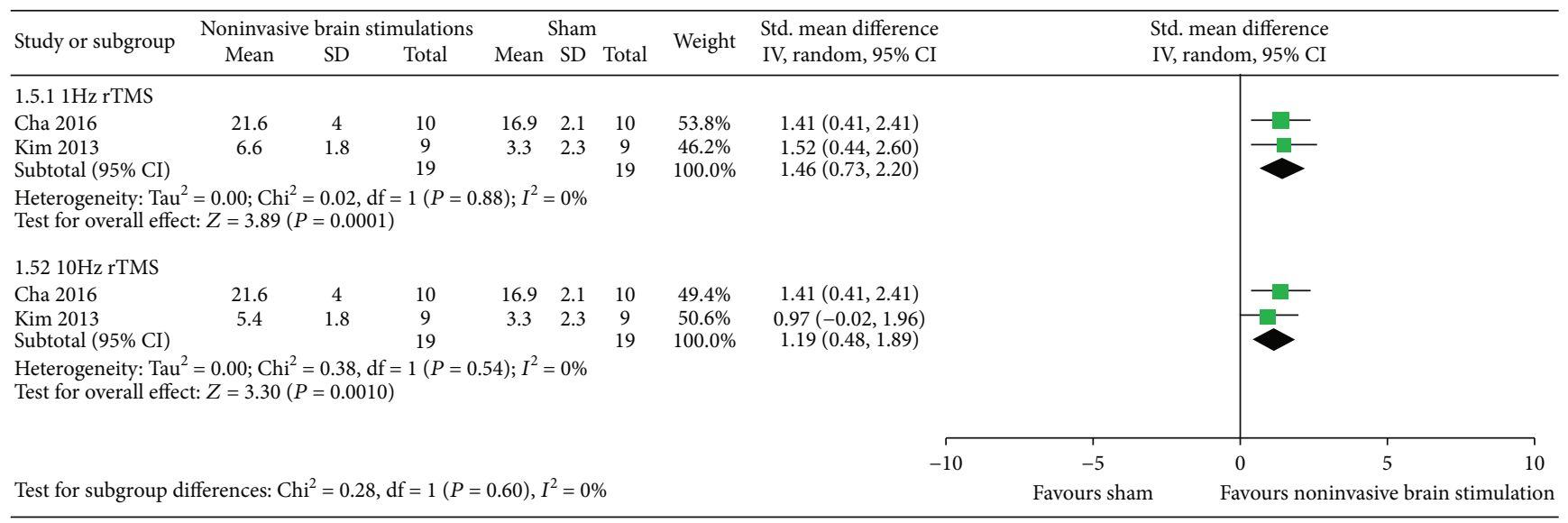

Figure 7: Meta-analysis of overall USN measured by the Motor-Free Visual Perception Test.

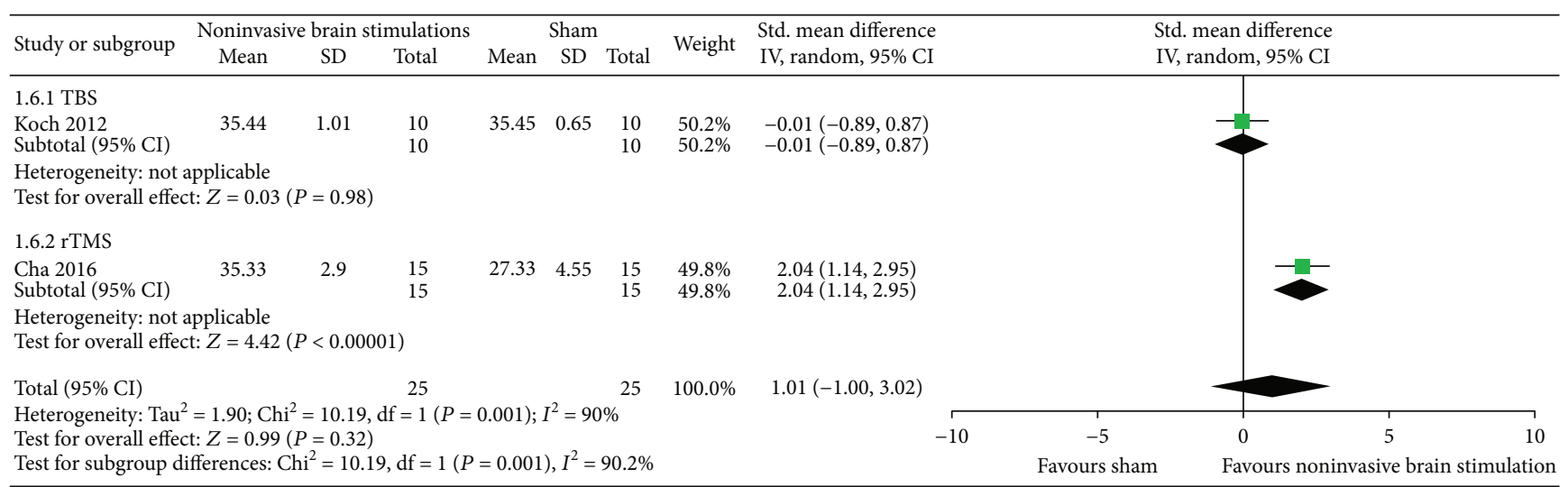

FIgURE 8: Meta-analysis of overall USN measured by Albert's test and the line crossing test.

pathological overactivation of the latter. This overactivation in the left depresses the neural activity by an increased inhibition on the right hemisphere, aggravating the perception. The rTMS can generate currents capable of depolarizing cortical neurons, and tDCS changes cortical activity by means of small electric currents and does not evoke action potentials. The tDCS has the advantage that the device is inexpensive, portable, and easy to use, but rTMS presented more activation of the neural network and induced a neuroplastic response for a long-term potentiation [65].

In three of four meta-analyses, rTMS was responsible for the improvement of overall USN, revealing that an electric current is an effective strategy for generating lasting promising effects in the brain. Unfortunately, we did not find any significant TBS or tDCS effects compared to sham procedures.

4.2. Strengths and Limitation. Strengths of our review include a comprehensive search; assessment of eligibility, risk of bias, and data abstraction independently and in duplicate; assessment of risk of bias that included a sensitivity analysis addressing loss to follow-up; and use of the GRADE approach for rating the certainty of evidence for each outcome (Table 3). Furthermore, there were no language restrictions, and translations of non-English trials were obtained whenever possible.

The primary limitation of our review is the low certainty consequent to study limitations. We identified a small number of RCTs with a modest number of participants resulting in wide confidence intervals. The total number of participants was relatively very low (RCTs $n=278$, non-RCTs $n=94$ ) due to the small sample sizes of individual trials, which led to downgrading the quality of evidence in some instances because underpowered trials are likely to have a greater degree of imprecision.

Moreover, selection bias and unblinding were substantial. Another limitation of this review was having an insufficient number of included studies to allow for the complete statistical analysis that we had planned. We were not able to assess publication bias because there were fewer than 10 eligible studies addressing the same outcome in a meta-analysis. We also planned to perform subgroup analyses according to the characteristics of stroke type, type of stimulation, type of frequency, and comparators (type of control intervention, i.e., pharmacological therapy versus nonpharmacological). However, we also were not able to conduct these analyses because they did not meet our minimal criteria, which was at least five studies available with at least two in each subgroup. 
Although this review presents several limitations, the issue is whether one should dismiss these results entirely or consider them bearing in mind the limitations. The latter represents our view of the matter.

4.3. Relation to Prior Work. The research question we investigated in our review has been addressed before from different perspectives using our population of interest but with a different intervention (i.e., pharmacological intervention) [7] or investigating either the intervention or the control arms explored in this review but with a different population (e.g., idiopathic Parkinson's disease (IPD) [48], panic disorder in adults [66], or amyotrophic lateral sclerosis or motor neuron disease) [13].

Two Cochrane reviews [21,49] evaluated the effect of tDCS in people after stroke but not in comparison with rTMS; instead, the authors compared tDCS with placebo, sham tDCS, no intervention, or conventional motor rehabilitation. The first review's [49] authors found evidence of effect regarding activities of daily living performance at the end of the intervention period and at the end of follow-up. However, the results did not persist in a sensitivity analysis including only trials of good methodological quality. In the second review [21], the authors found that there were no studies examining the effect of tDCS on cognition in stroke patients with aphasia.

Another Cochrane review [22] that addressed the use of rTMS compared to sham treatment or other conventional treatment for improving function after stroke revealed that rTMS treatment was not associated with improved activities of daily living, nor did it have a statistically significant effect on motor function.

Three additional Cochrane reviews also discussed the effects of both tDCS [48] and rTMS [13, 66] but in different populations-in Parkinsonism [48], in patients with amyotrophic lateral sclerosis or motor neuron disease [13], and in adults with panic disorder [66]. All reviews suffered from poor methodological quality, imprecision, and hence low confidence in the estimate of the true effect to draw a consistent conclusion on the effects of noninvasive brain stimulations.

4.4. Implications. Moderate-quality evidence shows that rTMS, at $1 \mathrm{~Hz}$, is more efficacious than sham for unilateral spatial neglect after stroke measured by Motor-Free Visual Perception Test. Furthermore, low-quality evidence also suggests a benefit of noninvasive brain stimulation, particularly with the use of rTMS, for overall USN measured by the line bisection test, Albert's test, and the line crossing test. Future trials should adhere to CONSORT guidelines to ensure clarity and reproducibility in the reporting of methods [67].

\section{Disclosure}

The funding agencies played no role in conducting the research or preparing the manuscript.

\section{Conflicts of Interest}

The authors have no conflicts of interest.

\section{Authors' Contributions}

Flávio Taira Kashiwagi, Regina El Dib, Adriana Bastos Conforto, Gustavo José Luvizutto, and Rodrigo Bazan conceived the review. Erica Aranha Suzumura, Taís Regina da Silva, and Fernanda Cristina Winckler undertake searches. Huda Gomaa, Juli Thomaz de Souza, and Nermeen Gawish screened search results. Flávio Taira Kashiwagi, Regina El Dib, and Gustavo José Luvizutto organized the retrieval of papers. Flávio Taira Kashiwagi, Huda Gomaa, Nermeen Gawish, Erica Aranha Suzumura, Taís Regina da Silva, and Rodrigo Bazan helped in screening retrieved papers against inclusion criteria. Flávio Taira Kashiwagi, Huda Gomaa, Nermeen Gawish, Erica Aranha Suzumura, Taís Regina da Silva, Fernanda Cristina Winckler, Juli Thomaz de Souza, and Rodrigo Bazan appraised the quality of papers. Flávio Taira Kashiwagi, Erica Aranha Suzumura, Taís Regina da Silva, and Fernanda Cristina Winckler extracted data from the papers. Flávio Taira Kashiwagi, Huda Gomaa, Nermeen Gawish, and Erica Aranha Suzumura helped in writing to the authors of papers for additional information. Flávio Taira Kashiwagi, Gustavo José Luvizutto, Juli Thomaz de Souza, and Nermeen Gawish provided additional data about the papers. Flávio Taira Kashiwagi, Erica Aranha Suzumura, Taís Regina da Silva, Nermeen Gawish, and Huda Gomaa obtained and screened the data of unpublished studies. Flávio Taira Kashiwagi, Regina El Dib, Gustavo José Luvizutto, and Rodrigo Bazan managed the data for the review. Flávio Taira Kashiwagi, Regina El Dib, Erica Aranha Suzumura, Gustavo José Luvizutto, and Rodrigo Bazan entered the data into Review Manager (RevMan). Regina El Dib, Gustavo José Luvizutto, Adriana Bastos Conforto, and Rodrigo Bazan analyzed the RevMan statistical data. Flávio Taira Kashiwagi, Huda Gomaa, Nermeen Gawish, Erica Aranha Suzumura, Adriana Bastos Conforto, Gustavo José Luvizutto, Regina El Dib, and Rodrigo Bazan interpreted the data. Flávio Taira Kashiwagi, Regina El Dib, Huda Gomaa, Nermeen Gawish, Erica Aranha Suzumura, Gustavo José Luvizutto, and Rodrigo Bazan made statistical inferences. Flávio Taira Kashiwagi, Gustavo José Luvizutto, Regina El Dib, Erica Aranha Suzumura, and Rodrigo Bazan wrote the review. Flávio Taira Kashiwagi, Huda Gomaa, Nermeen Gawish, Erica Aranha Suzumura, Gustavo José Luvizutto, Adriana Bastos Conforto, and Rodrigo Bazan take the responsibility for reading and checking the review before submission.

\section{Acknowledgments}

The authors would like to acknowledge São Paulo Research Foundation (FAPESP) (2015/14231-0) and National Council for Scientific and Technological Development (CNPq) (423924/2016-8) for the grants and financial support.

\section{References}

[1] R. Bonita, "Epidemiology of stroke," Lancet, vol. 339, no. 8789, pp. 342-344, 1992.

[2] E. C. Jauch, J. L. Saver, Adams HP Jr et al., "Guidelines for the early management of patients with acute ischemic stroke: a 
guideline for healthcare professionals from the American Heart Association/American Stroke Association," Stroke, vol. 44, no. 3, pp. 870-947, 2013.

[3] N. Gorgoraptis, Y. H. Mah, B. Machner et al., "The effects of the dopamine agonist rotigotine on hemispatial neglect following stroke," Brain, vol. 135, no. 8, pp. 2478-2491, 2012.

[4] P. Chen, K. Hreha, P. Fortis, K. M. Goedert, and A. M. Barrett, "Functional assessment of spatial neglect: a review of the Catherine Bergego scale and an introduction of the Kessler Foundation neglect assessment process," Topics in Stroke Rehabilitation, vol. 19, no. 5, pp. 423-435, 2012.

[5] S. P. Stone, P. W. Halligan, and R. J. Greenwood, "The incidence of neglect phenomena and related disorders in patients with an acute right or left hemisphere stroke," Age and Ageing, vol. 22, no. 1, pp. 46-52, 1993.

[6] M. Vanier, L. Gauthier, J. Lambert et al., "Evaluation of left visuospatial neglect: norms and discrimination power of two tests," Neuropsychology, vol. 4, no. 2, pp. 87-96, 1990.

[7] G. J. Luvizutto, R. Bazan, G. P. Braga et al., "Pharmacological interventions for unilateral spatial neglect after stroke," Cochrane Database of Systematic Reviews, vol. 1, no. 11, article CD010882, 2015.

[8] M. H. M. Tsang, K. H. Sze, and K. N. K. Fong, "Occupational therapy treatment with right half-field eye-patching for patients with subacute stroke and unilateral neglect: a randomised controlled trial," Disability and Rehabilitation, vol. 31, no. 8, pp. 630-637, 2009.

[9] H. Thieme, M. Bayn, M. Wurg, C. Zange, M. Pohl, and J. Behrens, "Mirror therapy for patients with severe arm paresis after stroke: a randomized controlled trial," Clinical Rehabilitation, vol. 27, no. 4, pp. 314-324, 2013.

[10] K. Mizuno, T. Tsuji, T. Takebayashi, T. Fujiwara, K. Hase, and M. Liu, "Prism adaptation therapy enhances rehabilitation of stroke patients with unilateral spatial neglect: a randomized, controlled trial," Neurorehabilitation and Neural Repair, vol. 25, no. 8, pp. 711-720, 2011.

[11] K. Polanowska, J. Seniów, E. Paprot, M. Leśniak, and A. Członkowska, "Left-hand somatosensory stimulation combined with visual scanning training in rehabilitation for post-stroke hemineglect: a randomised, double-blind study," Neuropsychological Rehabilitation, vol. 19, no. 3, pp. 364-382, 2009.

[12] A. Schröder, E. R. Wist, and V. Hömberg, “TENS and optokinetic stimulation in neglect therapy after cerebrovascular accident: a randomized controlled study," European Journal of Neurology, vol. 15, no. 9, pp. 922-927, 2008.

[13] J. Fang, M. Zhou, M. Yang, C. Zhu, L. He, and Cochrane Neuromuscular Group, "Repetitive transcranial magnetic stimulation for the treatment of amyotrophic lateral sclerosis or motor neuron disease," Cochrane Database of Systematic Reviews, vol. 1, no. 5, article CD008554, 2013.

[14] D. Cazzoli, R. M. Muri, R. Schumacher et al., "Theta burst stimulation reduces disability during the activities of daily living in spatial neglect," Brain, vol. 135, no. 11, pp. 3426-3439, 2012.

[15] J. Nakamura, Y. Kita, K. Ikuno, K. Kojima, Y. Okada, and K. Shomoto, "Influence of the stimulus parameters of galvanic vestibular stimulation on unilateral spatial neglect," Neuroreport, vol. 26, no. 8, pp. 462-466, 2015.

[16] M. F. Walker, A. Sunderland, J. Fletcher-Smith et al., "The DRESS trial: a feasibility randomized controlled trial of a neuropsychological approach to dressing therapy for stroke inpatients," Clinical Rehabilitation, vol. 26, no. 8, pp. 675$685,2012$.

[17] A. J. Turton, K. O'Leary, J. Gabb, R. Woodward, and I. D. Gilchrist, "A single blinded randomised controlled pilot trial of prism adaptation for improving self-care in stroke patients with neglect," Neuropsychological Rehabilitation, vol. 20, no. 2, pp. 180-196, 2010.

[18] M. A. Nitsche and W. Paulus, "Excitability changes induced in the human motor cortex by weak transcranial direct current stimulation," The Journal of Physiology, vol. 527, no. 3, pp. 633-639, 2000.

[19] L. J. Bindman, O. C. J. Lippold, and J. W. T. Redfearn, "The action of brief polarizing currents on the cerebral cortex of the rat (1) during current flow and (2) in the production of long-lasting after-effects," The Journal of Physiology, vol. 172, no. 3, pp. 369-382, 1964.

[20] D. A. Nowak, C. Grefkes, M. Ameli, and G. R. Fink, "Interhemispheric competition after stroke: brain stimulation to enhance recovery of function of the affected hand," Neurorehabilitation and Neural Repair, vol. 23, no. 7, pp. 641-656, 2009.

[21] B. Elsner, J. Kugler, M. Pohl, J. Mehrholz, and Cochrane Stroke Group, "Transcranial direct current stimulation (tDCS) for improving aphasia in patients with aphasia after stroke," Cochrane Database of Systematic Reviews, vol. 1, no. 5, article CD009760, 2015.

[22] Z. Hao, D. Wang, Y. Zeng, and M. Liu, "Repetitive transcranial magnetic stimulation for improving function after stroke," Cochrane Database of Systematic Reviews, vol. 1, no. 5, article CD008862, 2013.

[23] H. JPT, "Cochrane handbook for systematic reviews of interventions," Version 5.1.0 The Cochrane Collaboration2011, http://handbook-5-1.cochrane.org/.

[24] D. Moher, A. Liberati, J. Tetzlaff, D. G. Altman, and for the PRISMA Group, "Preferred reporting items for systematic reviews and meta-analyses: the PRISMA statement," $B M J$, vol. 339, article b2535, 2009.

[25] D. F. Stroup, J. A. Berlin, S. C. Morton et al., "Meta-analysis of observational studies in epidemiology: a proposal for reporting. Meta-analysis of Observational Studies in Epidemiology (MOOSE) group," Journal of the American Medical Association, vol. 283, no. 15, pp. 2008-2012, 2000.

[26] M. L. Albert, “A simple test of visual neglect," Neurology, vol. 23, no. 6, pp. 658-664, 1973.

[27] T. Schenkenberg, D. C. Bradford, and E. T. Ajax, "Line bisection and unilateral visual neglect in patients with neurologic impairment," Neurology, vol. 30, no. 5, pp. 509-517, 1980.

[28] P. W. Halligan, J. P. Burn, J. C. Marshall, and D. T. Wade, "Visuo-spatial neglect: qualitative differences and laterality of cerebral lesion," Journal of Neurology, Neurosurgery, and Psychiatry, vol. 55, no. 11, pp. 1060-1068, 1992.

[29] P. Azouvi, S. Olivier, G. de Montety, C. Samuel, A. LouisDreyfus, and L. Tesio, "Behavioral assessment of unilateral neglect: study of the psychometric properties of the Catherine Bergego scale," Archives of Physical Medicine and Rehabilitation, vol. 84, no. 1, pp. 51-57, 2003.

[30] B. Wilson, J. Cockburn, and P. Halligan, "Development of a behavioral test of visuospatial neglect," Archives of Physical Medicine and Rehabilitation, vol. 68, no. 2, pp. 98-102, 1987. 
[31] C. Cincura, O. M. Pontes-Neto, I. S. Neville et al., "Validation of the National Institutes of Health stroke scale, modified rankin scale and Barthel index in Brazil: the role of cultural adaptation and structured interviewing," Cerebrovascular Diseases, vol. 27, no. 2, pp. 119-122, 2009.

[32] V. Mathiowetz, G. Volland, N. Kashman, and K. Weber, "Adult norms for the box and block test of manual dexterity," American Journal of Occupational Therapy, vol. 39, no. 6, pp. 386-391, 1985.

[33] J. Sanford, J. Moreland, L. R. Swanson, P. W. Stratford, and C. Gowland, "Reliability of the Fugl-Meyer assessment for testing motor performance in patients following stroke," Physical Therapy, vol. 73, no. 7, pp. 447-454, 1993.

[34] J. M. Morse, C. Black, K. Oberle, and P. Donahue, "A prospective study to identify the fall-prone patient," Social Science \& Medicine, vol. 28, no. 1, pp. 81-86, 1989.

[35] A. L. Hendrich, P. S. Bender, and A. Nyhuis, "Validation of the Hendrich II fall risk model: a large concurrent case/control study of hospitalized patients," Applied Nursing Research, vol. 16, no. 1, pp. 9-21, 2003.

[36] H. F. Mao, I. P. Hsueh, P. F. Tang, C. F. Sheu, and C. L. Hsieh, "Analysis and comparison of the psychometric properties of three balance measures for stroke patients," Stroke, vol. 33, no. 4, pp. 1022-1027, 2002.

[37] I. Aben, F. Verhey, R. Lousberg, J. Lodder, and A. Honig, "Validity of the Beck depression inventory, hospital anxiety and depression scale, SCL-90, and Hamilton depression rating scale as screening instruments for depression in stroke patients," Psychosomatics, vol. 43, no. 5, pp. 386-393, 2002.

[38] A. Lerdal and A. Kottorp, "Psychometric properties of the fatigue severity scale-Rasch analyses of individual responses in a Norwegian stroke cohort," International Journal of Nursing Studies, vol. 48, no. 10, pp. 1258-1265, 2011.

[39] J. P. T. Higgins, D. G. Altman, P. C. Gotzsche et al., "The Cochrane collaboration's tool for assessing risk of bias in randomised trials," $B M J$, vol. 343, no. oct18 2, p. d5928, 2011.

[40] G. H. Guyatt and J. W. Busse, "Modification of Cochrane tool to assess risk of bias in randomized trials," http://distillercer .com/resources/.

[41] G. H. Guyatt, A. D. Oxman, G. Vist et al., "GRADE guidelines: 4. Rating the quality of evidence-study limitations (risk of bias)," Journal of Clinical Epidemiology, vol. 64, no. 4, pp. 407-415, 2011.

[42] G. H. Guyatt, A. D. Oxman, G. Vist et al., "GRADE guidelines: 5. Rating the quality of evidence-study limitations (risk of bias)," Journal of Clinical Epidemiology, vol. 64, no. 4, pp. 407-415, 2011.

[43] G. H. Guyatt, A. D. Oxman, R. Kunz et al., "GRADE guidelines 6. Rating the quality of evidence-imprecision," Journal of Clinical Epidemiology, vol. 64, no. 12, pp. 1283-1293, 2011.

[44] G. H. Guyatt, A. D. Oxman, R. Kunz et al., "GRADE guidelines: 7. Rating the quality of evidence-inconsistency," Journal of Clinical Epidemiology, vol. 64, no. 12, pp. 12941302, 2011.

[45] G. H. Guyatt, A. D. Oxman, R. Kunz et al., "GRADE guidelines: 8. Rating the quality of evidence-indirectness," Journal of Clinical Epidemiology, vol. 64, no. 12, pp. 1303-1310, 2011.

[46] G. H. Guyatt, A. D. Oxman, V. Montori et al., "GRADE guidelines: 5. Rating the quality of evidence-publication bias,"
Journal of Clinical Epidemiology, vol. 64, no. 12, pp. 12771282, 2011.

[47] The Cochrane Collaboration, Review Manager (RevMan). 5.3., The Nordic Cochrane Centre, The Cochrane Collaboration, Copenhagen, Denmark, 2011.

[48] B. Elsner, J. Kugler, M. Pohl, and J. Mehrholz, "Transcranial direct current stimulation (tDCS) for idiopathic Parkinson's disease," Cochrane Database of Systematic Reviews, vol. 1, no. 7, article CD010916, 2016.

[49] B. Elsner, J. Kugler, M. Pohl, and J. Mehrholz, "Transcranial direct current stimulation (tDCS) for improving activities of daily living, and physical and cognitive functioning, in people after stroke," Cochrane Database of Systematic Reviews, vol. 1, no. 3, article CD009645, 2016.

[50] L. Cao, W. Fu, Y. Zhang et al., "Intermittent $\theta$ burst stimulation modulates resting-state functional connectivity in the attention network and promotes behavioral recovery in patients with visual spatial neglect," Neuroreport, vol. 27, no. 17, pp. 1261-1265, 2016.

[51] H. G. Cha and M. K. Kim, "Effects of repetitive transcranial magnetic stimulation on arm function and decreasing unilateral spatial neglect in subacute stroke: a randomized controlled trial," Clinical Rehabilitation, vol. 30, no. 7, pp. 649-656, 2016.

[52] W. Fu, W. Song, Y. Zhang et al., "Long-term effects of continuous theta-burst stimulation in visuospatial neglect," The Journal of International Medical Research, vol. 43, no. 2, pp. 196-203, 2015.

[53] B. R. Kim, M. H. Chun, D. Y. Kim, and S. J. Lee, "Effect of highand low-frequency repetitive transcranial magnetic stimulation on visuospatial neglect in patients with acute stroke: a double-blind, sham-controlled trial," Archives of Physical Medicine and Rehabilitation, vol. 94, no. 5, pp. 803-807, 2013.

[54] G. Koch, S. Bonni, V. Giacobbe et al., "Theta-burst stimulation of the left hemisphere accelerates recovery of hemispatial neglect," Neurology, vol. 78, no. 1, pp. 24-30, 2012.

[55] W. Yang, T. T. Liu, X. B. Song et al., "Comparison of different stimulation parameters of repetitive transcranial magnetic stimulation for unilateral spatial neglect in stroke patients," Journal of the Neurological Sciences, vol. 359, no. 1-2, pp. 219-225, 2015.

[56] M. Smit, D. J. L. G. Schutter, T. C. W. Nijboer et al., “Transcranial direct current stimulation to the parietal cortex in hemispatial neglect: a feasibility study," Neuropsychologia, vol. 74, pp. 152-161, 2015.

[57] H. Sunwoo, Y. H. Kim, W. H. Chang, S. Noh, E. J. Kim, and M. H. Ko, "Effects of dual transcranial direct current stimulation on post-stroke unilateral visuospatial neglect," Neuroscience Letters, vol. 554, pp. 94-98, 2013.

[58] M. H. Ko, S. H. Han, S. H. Park, J. H. Seo, and Y. H. Kim, "Improvement of visual scanning after DC brain polarization of parietal cortex in stroke patients with spatial neglect," Neuroscience Letters, vol. 448, no. 2, pp. 171-174, 2008.

[59] G. Koch, S. Bonnì, V. Giacobbe et al., "Theta-burst stimulation of the left hemisphere accelerates recovery of hemispatial neglect," Neurology, vol. 78, no. 1, pp. 24-30, 2012.

[60] W. Fu, L. Cao, Y. Zhang et al., "Continuous theta-burst stimulation may improve visuospatial neglect via modulating the attention network: a randomized controlled study," Topics in Stroke Rehabilitation, vol. 24, no. 4, pp. 236-241, 2017.

[61] D. Cazzoli, C. R. Rosenthal, C. Kennard et al., "Theta burst stimulation improves overt visual search in spatial neglect 
independently of attentional load," Cortex, vol. 73, pp. 317329, 2015.

[62] S. Hopfner, D. Cazzoli, R. M. Müri et al., "Enhancing treatment effects by combining continuous theta burst stimulation with smooth pursuit training," Neuropsychologia, vol. 74, pp. 145-151, 2015.

[63] S. Agosta, F. Herpich, G. Miceli, F. Ferraro, and L. Battelli, "Contralesional rTMS relieves visual extinction in chronic stroke," Neuropsychologia, vol. 62, pp. 269-276, 2014.

[64] E. Làdavas, S. Giulietti, A. Avenanti et al., "A-tDCS on the ipsilesional parietal cortex boosts the effects of prism adaptation treatment in neglect," Restorative Neurology and Neuroscience, vol. 33, no. 5, pp. 647-662, 2015.

[65] R. M. Müri, D. Cazzoli, T. Nef, U. P. Mosimann, S. Hopfner, and T. Nyffeler, "Non-invasive brain stimulation in neglect rehabilitation: an update," Frontiers in Human Neuroscience, vol. 7, p. 248, 2013.

[66] H. Li, J. Wang, C. Li, and Z. Xiao, "Repetitive transcranial magnetic stimulation (rTMS) for panic disorder in adults," Cochrane Database of Systematic Reviews, vol. 1, no. 9, article CD009083, 2014.

[67] F. T. Kashiwagi, Neon-invasive brain stimulations for unilateral spatial neglect after Strokestroke: a systematic review and meta-analysis of randomized and non-randomized controlled Trials. Dissertation [M.S. thesis], Botucatu Medical School, UNESP, 2003. 


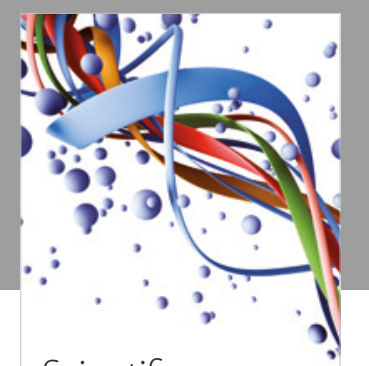

Scientifica
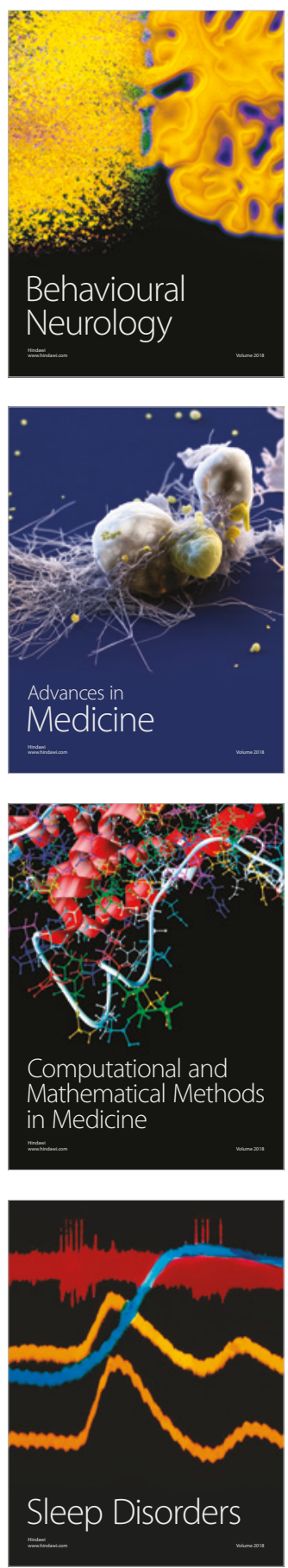

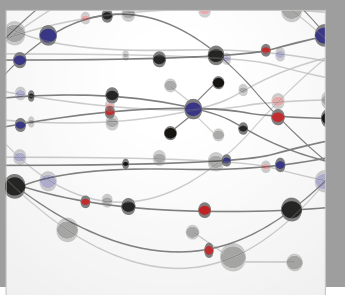

The Scientific World Journal

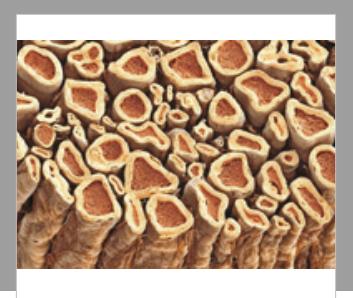

Case Reports in

Neurological Medicine

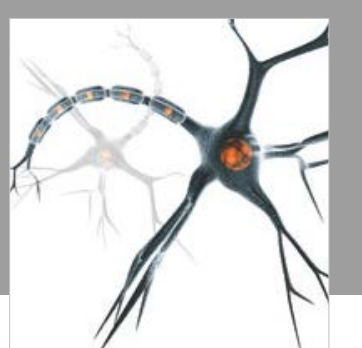

Neural Plasticity

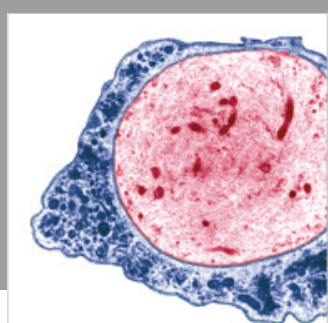

Multiple Sclerosis

International

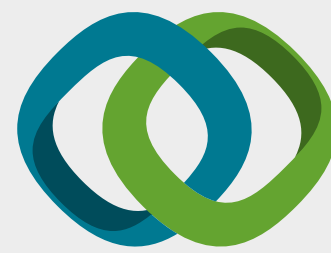

Hindawi

Submit your manuscripts at

www.hindawi.com
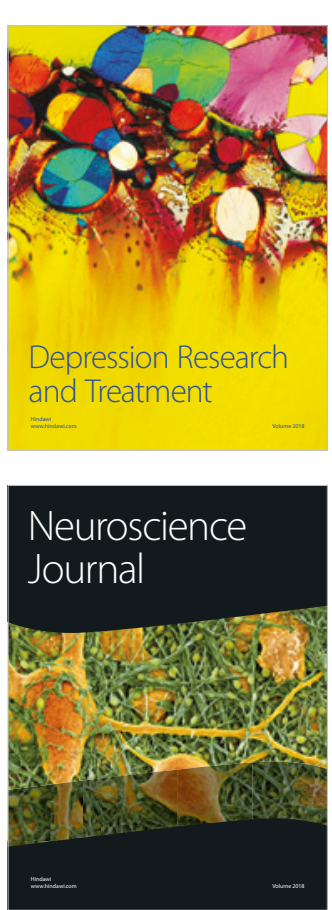

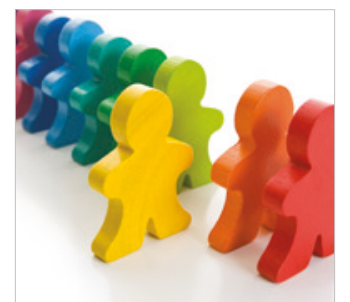

Autism

Research and Treatment
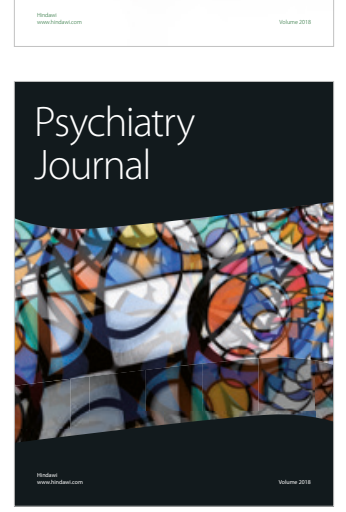
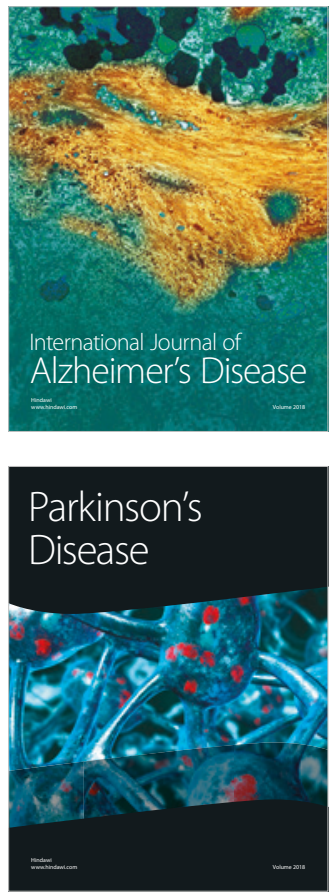
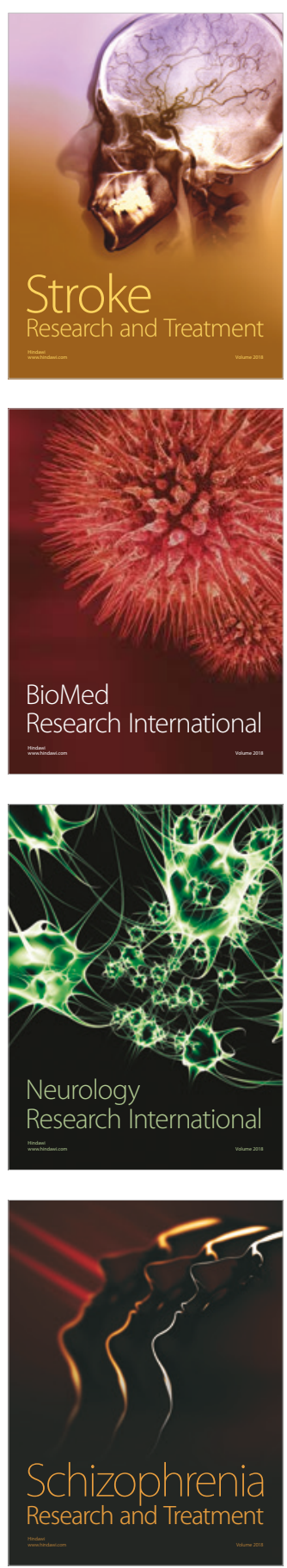